\title{
Transcriptomic responses to diet quality and viral infection in Apis mellifera
}

\author{
Lindsay Rutter ${ }^{1}$, Jimena Carrillo-Tripp ${ }^{2}$, Bryony C. Bonning ${ }^{3}$, Dianne Cook ${ }^{4}$, Amy L. Toth 5 5,6 \\ and Adam G. Dolezal ${ }^{* *}$ (1)
}

\begin{abstract}
Background: Parts of Europe and the United States have witnessed dramatic losses in commercially managed honey bees over the past decade to what is considered an unsustainable extent. The large-scale loss of bees has considerable implications for the agricultural economy because bees are one of the leading pollinators of numerous crops. Bee declines have been associated with several interactive factors. Recent studies suggest nutritional and pathogen stress can interactively contribute to bee physiological declines, but the molecular mechanisms underlying interactive effects remain unknown. In this study, we provide insight into this question by using RNA-sequencing to examine how monofloral diets and Israeli acute paralysis virus inoculation influence gene expression patterns in bees. Results: We found a considerable nutritional response, with almost 2000 transcripts changing with diet quality. The majority of these genes were over-represented for nutrient signaling (insulin resistance) and immune response (Notch signaling and JaK-STAT pathways). In our experimental conditions, the transcriptomic response to viral infection was fairly limited. We only found 43 transcripts to be differentially expressed, some with known immune functions (argonaute-2), transcriptional regulation, and muscle contraction. We created contrasts to explore whether protective mechanisms of good diet were due to direct effects on immune function (resistance) or indirect effects on energy availability (tolerance). A similar number of resistance and tolerance candidate differentially expressed genes were found, suggesting both processes may play significant roles in dietary buffering from pathogen infection.

Conclusions: Through transcriptional contrasts and functional enrichment analysis, we contribute to our understanding of the mechanisms underlying feedbacks between nutrition and disease in bees. We also show that comparing results derived from combined analyses across multiple RNA-seq studies may allow researchers to identify transcriptomic patterns in bees that are concurrently less artificial and less noisy. This work underlines the merits of using data visualization techniques and multiple datasets to interpret RNA-sequencing studies.
\end{abstract}

Keywords: Honey bee, RNA-sequencing, Israeli acute paralysis virus, Monofloral pollen, Visualization

\section{Background}

Managed honey bees have undergone health declines in the United States and parts of Europe over the past decade [1-3], with annual mortality rates exceeding what beekeepers consider sustainable $[4,5]$. More than 70 percent of major global food crops (including fruits, vegetables, and nuts) at least benefit from pollination, and yearly insect pollination services are valued worldwide at $\$ 175$ billion [6]. As honey bees are largely considered to be the leading pollinator of numerous crops, their

*Correspondence: adolezal@illinois.edu

${ }^{7}$ Department of Entomology, University of Illinois at Urbana-Champaign, Urbana 61801, IL, USA

Full list of author information is available at the end of the article marked loss has considerable implications for agricultural sustainability [7].

Honey bee declines have been associated with several factors, including pesticide use, parasites, pathogens, habitat loss, and poor nutrition $[8,9]$. Researchers generally agree that these stressors do not act in isolation; instead, they appear to influence the large-scale loss of honey bees in an interactive fashion as the environment changes [10]. Nutrition and viral infection are two factors that pose heightened dangers to honey bee health in response to recent environmental changes. Interactions between nutrition and viral infection may create feedbacks that impact bee health through several mechanisms $[11,12]$.

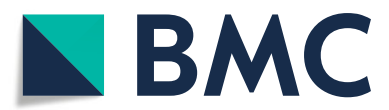

(c) The Author(s). 2019 Open Access This article is distributed under the terms of the Creative Commons Attribution 4.0 International License (http://creativecommons.org/licenses/by/4.0/), which permits unrestricted use, distribution, and reproduction in any medium, provided you give appropriate credit to the original author(s) and the source, provide a link to the Creative Commons license, and indicate if changes were made. The Creative Commons Public Domain Dedication waiver (http://creativecommons.org/publicdomain/zero/1.0/) applies to the data made available in this article, unless otherwise stated. 
Pollen is a main source of nutrition (including proteins, amino acids, lipids, sterols, starch, vitamins, and minerals) in honey bees $[13,14]$. At the individual level, pollen supplies most of the nutrients necessary for physiological development [15] and is believed to have considerable impact on longevity [16]. At the colony level, pollen enables young workers to produce jelly, which then nourishes larvae, drones, older workers, and the queen $[17,18]$. Various environmental changes (including urbanization and monoculture crop production) have significantly altered the nutritional profile available to honey bees. In particular, honey bees are confronted with a less diverse selection of pollen, which is of concern because mixed-pollen (polyfloral) diets are generally considered healthier than single-pollen (monofloral) diets [19-21]. Reported colony mortality rates are higher in developed land areas compared to undeveloped land areas [22], and beekeepers rank poor nutrition as one of the main reasons for colony losses [23]. Understanding how low diversity diets (i.e. monofloral diets) affect honey bee health will be crucial to resolve problems that may arise as agriculture continues to intensify throughout the world [24, 25]. Indeed, differing qualities of monofloral diets have been shown to affect nurse bee physiology and tolerance to parasites [26].

Viral infection was considered a comparatively minor problem in honey bees until the last century when the ectoparasitic varroa mite (Varroa destructor) spread worldwide [27-29]. This mite feeds on honey bee hemolymph and/or fat body tissue [30,31], and is believed to decrease lipid and glycogen reserves and reduce protein synthesis in bees [32]. Additionally, it transmits multiple viruses and supports replication of some viruses [33-36]. More than 20 honey bee viruses have been identified [37]. One of these viruses that has been linked to honey bee decline is Israeli acute paralysis virus (IAPV), a positive-sense RNA virus of the family Dicistroviridae [38]. IAPV infection causes shivering wings, decreased locomotion, muscle spasms, paralysis, and high premature death percentages in caged infected adult honey bees [39]. IAPV has demonstrated higher infectious capacity [40] and is more prevalent in colonies that do not survive the winter [41].

Although there is growing interest in how viruses and diet quality affect the health and sustainability of honey bees, as well as a recognition that such factors might operate interactively, there are only a small number of experimental studies thus far directed toward elucidating the interactive effects of these two factors in honey bees [42-46]. We recently used laboratory cages and nucleus hive experiments to investigate the health effects of these two factors, and our results show the importance of the combined effects of both diet quality and virus infection. Specifically, ingestion by honey bees of high quality pollen is able to mitigate virus-induced mortality to the level of diverse, polyfloral pollen [11].

Following up on these findings, we now aim to understand the corresponding underlying mechanisms by which high quality diets protect bees from virus-induced mortality. For example, it is not known whether the protective effect of good diet is due to direct, specific effects on immune function that reduce the pathogen load of the host (resistance, [47]) or if it is due to indirect effects of good nutrition on the ability of the host to withstand pathogen impacts without affecting pathogen load (tolerance, [47]). Transcriptomics is one means to better understand the mechanistic underpinnings of dietary and viral effects on honey bee health. Transcriptomic analysis can help us identify 1) the genomic scale of transcriptomic response to diet and virus infection, 2) whether these factors interact in an additive or synergistic way on transcriptome function, and 3) the types of pathways affected by diet quality and viral infection, which can help us generate candidate gene lists to further investigate the relative roles of tolerance and resistance. This information, heretofore lacking in the literature, can help us better understand how good nutrition may be able to serve as a "buffer" against other stressors [12].

There are only a small number of published experiments examining gene expression patterns related to diet effects [48] and virus infection effects [49-53] in honey bees, but there have been several such studies in model organisms. Model insect studies can inform studies of honey bee transcriptomic responses, using functional inference of as-of-yet uncharacterized honey bee genes based on orthology to Drosophila and other model organisms. Previous Drosophila studies that examined various diet effects have found gene expression changes related to immunity, metabolism, cell cycle activity, DNA binding, transcription, and insulin signaling [48, 54-56]. While similar transcriptomic studies have been limited in honey bees, one study found that pollen nutrition upregulates genes involved in macromolecule metabolism, longevity, and the insulin/TOR pathway $[48,51]$. Previous transcriptomic studies have identified genes serving as links between metabolism and antiviral defense in honey bees $[57,58]$; see [59] for an overview. Numerous studies on the transcriptomic effects of virus infection in model insect organisms have shown that RNA silencing, transcriptional pausing, Toll pathways, IMD pathways, JAK/STAT pathways, and Toll-7 autophagy pathways play substantial roles in virus-host systems [60, 61]. Studies of virus-bee systems have revealed some of the antiviral defense pathways known in model organisms are conserved and also related to bee antiviral immune responses [62].

To our knowledge, there are few to no studies investigating honey bee gene expression patterns specifically related to monofloral diets, and few studies investigating 
honey bee gene expression patterns related to the combined effects of diet in any broad sense and viral inoculation in any broad sense [45]. In this study, we examine how monofloral diets and viral inoculation influence gene expression patterns in honey bees by focusing on four treatment groups (low quality diet without IAPV inoculation, high quality diet without IAPV inoculation, low quality diet with IAPV inoculation, and high quality diet with IAPV inoculation). For our diet factor, we examined two monofloral pollen diets, rockrose (Cistus sp.) and chestnut (Castanea sp.). Rockrose pollen is generally considered less nutritious than chestnut pollen because it contains smaller amounts of protein, amino acids, antioxidants, calcium, and iron [11,26]. For specific quantitative differences between these two pollen groups, please see [26]. Throughout this paper, we refer to our four treatment groups as "NR" (non-inoculation and low quality rockrose pollen), "NC" (non-inoculation and high quality chestnut pollen), "VR" (IAPV inoculation and low quality rockrose pollen), and "VC" (IAPV inoculation and high quality chestnut pollen). We conduct RNA-sequencing analysis on a randomly selected subset of the honey bees we used in our previous study (as is further described in our methods section). We then examine pairwise combinations of treatment groups, the main effect of monofloral diet, the main effect of IAPV exposure, and the combined effect of the two factors on gene expression patterns.

Because RNA-seq data can be noisy and subject to high levels of inter-experiment variation, we further sought to validate our transcriptomic data via comparison to a previous RNA-seq study on honey bee responses to viral infection. To do this, we compare the main effect of IAPV exposure in our dataset to that obtained in a previous study conducted by Galbraith and colleagues [49]. While our study examines honey bees derived from naturallymated queens, the Galbraith study examined honey bees derived from single-drone inseminated queens. As a consequence, intracolony worker relatedness in our study is around 25\%, compared to $75 \%$ in the Galbraith study [63]. Genetic diversity of bees in our study was thus higher than the Galbraith study, and was likely to be especially high because we sampled from 15 different colonies, i.e. from 15 different, naturally-mated queens. We should therefore expect that the Galbraith study may generate data with higher signal-to-noise ratios than our data due to lower genetic variation between its replicates. At the same time, our honey bees will be more likely to display the health benefits gained from increased genotypic variance within colonies, including decreased parasitic load [64], increased tolerance to environmental changes [65], and increased colony performance [66, 67]. Given that honey bees are naturally very polyandrous [68], our naturally-mated honey bees may also reflect more realistic environmental and genetic conditions. To achieve this comparison, we use visualization techniques to assess the signal:to:noise ratio between these two datasets, and differential gene expression (DEG) analyses to determine any significantly overlapping genes of interest between these two datasets. As RNA-sequencing data can be biased [69-71], this comparison allowed us to characterize how repeatable and robust our RNA-sequencing results were in comparison to previous studies. It also allowed us to shine light on how experimental designs that control genetic variability to different extents might affect the resulting gene expression data in honey bees. We suggest that in-depth data visualization approaches (including scatterplot matrices, parallel coordinate plots, and litre plots from the bigPint software package [72]) can be useful for cross-study comparisons and validation of noisy RNA-sequencing data in the future.

\section{Methods}

\section{Mortality and virus titers}

Details of the procedures we used to prepare virus inoculum, infect and feed caged honey bees, and quantify IAPV can be reviewed in our previous work [11, 40]. In brief, our virus inoculum was prepared by injection of infectious virus particles (derived from infected adults) into whiteeyed honey bee pupae; these pupae were then homogenized and virus particles enriched and resuspended. This inoculum was then characterized for presence of acute bee paralysis virus, black queen cell virus, deformed wing virus (DWV), IAPV, Kashmir bee virus, and sacbrood bee virus (SBV). Experimental infection tests of adult bees and honey bee cell cultures [40] showed that only IAPV is amplified in adult bees. To infect caged bees for these experiments, newly emerged bees from 15 healthy colonies at the Iowa State University research apiary were homogeneously mixed, then counted into clear acrylic cages in groups of 35 bees per cage. Cages were then presented with open feeders containing 30\% sucrose solution (control) or $30 \%$ sucrose solution containing a 1:1000 dilution of viral inoculum (treatment). Dietary treatments were then added (described below). To quantify virus titers, two live bees were randomly sampled at $36 \mathrm{hpi}$ from each of 9-10 randomly selected cages. Virus levels were then measured via RT-qPCR and quantified against a standard curve, identically to methods described in $[11,40]$.

A linear mixed effects model was used to relate the mortality rates and IAPV titers to the main and interaction effects of the diet and virus factors. The model was fit to the data by restricted maximum likelihood (REML) using the "lme" function in the R package "nlme". A random (intercept) effect for experimental setup was included in the model. Post-hoc pairwise comparisons of the four (diet and virus combination) treatment groups were performed and Benjamini-Hochberg adjusted 
$p$-values were calculated to limit familywise Type I error rates [73].

\section{Design of two-factor experiment}

For our nutrition factor, we examined two monofloral pollen diets, rockrose (Cistus sp.) and chestnut (Castanea sp.). Rockrose pollen is generally considered less nutritious than chestnut pollen due to its lower levels of protein, amino acids, antioxidants, calcium, and iron $[11,26]$. For our virus factor, one level contained bees that were inoculated with IAPV and another level contained bees that were not inoculated with IAPV. This experimental design resulted in four treatment groups (NR: low quality rockrose pollen without IAPV exposure; NC: high quality chestnut pollen without IAPV exposure; VR: low quality rockrose pollen with IAPV exposure; VC: high quality chestnut pollen with IAPV exposure) that allowed us to assess main effects and interactive effects between diet quality and IAPV infection in honey bees.

There are several reasons why our design focused only on diet quality (monofloral diets) as opposed to diet diversity (monofloral diets versus polyfloral diets). First, when assessing diet diversity, a sugar diet is often used as a control. However, such an experimental design does not reflect real-world conditions for honey bees as they rarely face a total lack of pollen [26]. Moreover, younger larvae tend to be fed pollen diets, whereas older larvae tend to be fed nectar diets. By focusing on pollen diets, our study design reflects natural diet conditions for larvae of a specific age category [74]. Second, in studies that compared honey bee health using monofloral and polyfloral diets at the same time, if the polyfloral diet and one of the high quality monofloral diets both exhibited similarly beneficial effects, then it was difficult for the authors to assess if the polyfloral diet was better than most of the monofloral diets because of its diversity or because it contained as a subset the high quality monofloral diet [26]. Third, as was previously mentioned, honey bees are now confronted with less diverse sources of pollen. As a result, there is a need to better understand how monofloral diets affect honey bee health.

\section{RNA extraction}

Fifteen cages per treatment were originally produced for monitoring of mortality. From these, six live honey bees were randomly selected from each cage $36 \mathrm{~h}$ post inoculation and placed into tubes [40]. In summary, 8 samples (representing two bees each) were sequenced per experimental condition (i.e., 32 samples sequenced). Tubes were kept on dry ice and then transferred into a $-80 \mathrm{C}$ freezer until processing. From the fifteen possible cages, eight were randomly selected for RNA-sequencing. From these eight cages, two of the honey bees per cage were randomly selected from the original six live honey bees per cage. These two bees were combined to form a pooled sample representing the cage. Whole body RNA from each pool was extracted using Qiagen RNeasy MiniKit followed by Qiagen DNase treatment. Samples were suspended in water to $200-400 \mathrm{ng} / \mu \mathrm{l}$. All samples were then tested on a Bioanalyzer at the Iowa State University DNA Facility to ensure quality (RIN $>8$ ).

\section{Gene expression}

Samples were sequenced starting on January 14, 2016 at the Iowa State University DNA Facility (Platform: Illumina HiSeq Sequencing 2500 in rapid run mode; Category: Single End 100 cycle sequencing). A standard Illumina mRNA library was prepared by the DNA facility. Reads were aligned to the BeeBase Version 3.2 genome [75] from the Hymenoptera Genome Database [76] using the programs GMAP and GSNAP [77]. There were four lanes of sequencing with 24 samples per lane. Each sample was run twice. Approximately $75-90 \%$ of reads were mapped to the honey bee genome. Each lane produced around 13 million single-end 100 basepair reads.

We tested all six pairwise combinations of treatments for DEGs (pairwise DEGs: NR versus NC, NR versus $\mathrm{VR}, \mathrm{NR}$ versus $\mathrm{VC}, \mathrm{NC}$ versus $\mathrm{VR}, \mathrm{NC}$ versus $\mathrm{VC}$, and $\mathrm{VR}$ versus $\mathrm{VC}$ ). We also tested the diet main effect (diet DEGs), virus main effect (virus DEGs), and interaction term for DEGs (interaction DEGs). We then also tested for virus main effect DEGs (virus DEGs) in public data derived from a previous study exploring the gene expression of IAPV virus infection in honey bees [49]. We tested each DEG analysis using recommended parameters with DESeq2 [78], edgeR [79], and LimmaVoom [80]. For our DEG analysis, we used R software version 3.3.3 [81]. In all cases, we used a false discovery rate (FDR) threshold of 0.05 [82]. Fisher's exact test was used to determine significant overlaps between DEG sets (whether from the same dataset but across different analysis pipelines or from different datasets across the same analysis pipelines). The eulerr shiny application was used to construct Venn diagram overlap images [83]. In the end, we focused on the DEG results from DESeq2 [78] as this pipeline was also used in the Galbraith study [49]. We used the independent filtering process built into the DESeq2 software that mitigates multiple comparison corrections on genes with no power rather than defining one filtering threshold.

\section{Comparison to prior studies on transcriptomic response to viral infection}

We compare the main effect of IAPV exposure in our dataset to that obtained in a previous study conducted by Galbraith and colleagues [49] who also addressed honey bee transcriptomic responses to virus infection. 
We applied the same downstream bioinformatics analyses between our count table and the count table provided in the Galbraith study. When we applied our bioinformatics pipeline to the Galbraith count table, we obtained different differential expression counts compared to the results published in the Galbraith study. However, there was substantial overlap and we considered this justification to use the differential expression list we obtained in order to keep the downstream bioinformatics analyses as similar as possible between the two datasets (Additional file 17).

We used honey bees from naturally-mated colonies, whereas Galbraith et al. [49] used honey bees from singledrone colonies. In light of this, we should expect the Galbraith et al. dataset to contain lower genetic variation between its replicates and higher signal-to-noise ratios than our dataset. We use visualization techniques to assess the signal-to-noise ratio between these two datasets, and differential gene expression (DEG) analyses to determine any significantly overlapping genes of interest between these two datasets.

\section{Visualization}

We used an array of visualization tools as part of our analysis. We used the PCA plot [84] from the DESeq2 package, a well-known and established tool. Along with that, we used lesser-known multivariate visualization tools from our R package called bigPint [72]. Specifically, we used parallel coordinate plots [85], scatterplot matrices [86], and litre plots (which we recently developed based on "replicate line plots" [87]) to assess the variability between the replicates and the treatments in our data. We also used these plotting techniques to assess for normalization problems and other common problems in RNAsequencing analysis pipelines [87].

Furthermore, we used statistical graphics to better understand patterns in our DEGs. However, in cases of large DEG lists, these visualization tools had overplotting problems (where multiple objects are drawn on top of one another, making it impossible to detect individual values). To remedy this problem, we first standardized each DEG to have a mean of zero and standard deviation of unity for its read counts across its samples [88, 89]. Then, we performed hierarchical clustering on the standardized DEGs using Ward's linkage. This process divided large DEG lists into smaller clusters of similar patterns, which allowed us to more efficiently visualize the different types of patterns within large DEG lists (see Figs. 3 and 4 for examples).

\section{Gene ontology}

DEGs were uploaded as a background list to DAVID Bioinformatics Resources 6.7 [90, 91]. The overrepresented gene ontology (GO) terms of DEGs were determined using the BEEBASE_ID identifier option (honey bee gene model) in the DAVID software. To fine-tune the GO term list, only terms correlating to Biological Processes were considered. The refined GO term list was then imported into REVIGO [92], which uses semantic similarity measures to cluster long lists of GO terms.

\section{Probing tolerance versus resistance}

To investigate whether the protective effect of good diet is due to direct, specific effects on immune function (resistance), or if it is due to indirect effects of good nutrition on energy availability and vigor (tolerance), we created contrasts of interest (Table 2). In particular, we assigned "resistance candidate DEGs" to be the ones that were upregulated in the chestnut group within the virus inoculated bees but not upregulated in the chestnut group within the non-inoculated bees. Our interpretation of these genes is that they represent those that are only activated in inoculated bees that are fed a high quality diet. We also assigned "tolerance candidate DEGs" to be the ones that were upregulated in the chestnut group for both the virus inoculated bees and non-inoculated bees. Our interpretation of these genes is that they represent those that are constitutively activated in bees fed a high quality diet, regardless of whether they are experiencing infection or not. We then determined how many genes fell into these two categories and analyzed their GO functions.

\section{Post hoc analysis}

We found considerable noisiness in our data and saw, through gene-level visualizations, that our DEGs contained outliers and inconsistent replicates. Hence, we wanted to explore whether our DEG read counts correlated with pathogen response metrics, including IAPV titers, SBV titers (also present in our inoculum [11, $40]$ ), and mortality rates. We explored correlation with SBV because our inoculum [40] does contain SBV, and bees from both inoculated and non-inoculated groups do exhibit detectable SBV titers. For this process, we considered virus main effect DEGs (Fig. 4), "tolerance candidate" DEGs (Additional file 15), and "resistance candidate" DEGs (Additional file 16). For each DEG in each cluster, we calculated a coefficient of determination (R-squared) value to estimate the correlation between its raw read counts and the pathogen response metrics across its 24 samples. We then used the KruskalWallis test to determine if the distribution of the Rsquared values in any of the DEG clusters significantly differed from those in the non-DEG genes (the rest of the data). As there were four clusters for each of the nine combinations of DEG lists ("tolerance" candidate DEGs, "resistance" candidate DEGs, and virus-related DEGs) and pathogen response measurements (IAPV titer, SBV titer, and mortality rate), this process resulted in 36 statistical tests. 


\section{Results}

\section{Mortality and virus titers}

We reanalyzed our previously published dataset with a subset that focuses on diet quality and is more relevant to the current study. We show the data subset here to inform the RNA-sequencing comparison because we reduced the number of treatments from the original published data (from eight to four) [11] as a means to focus on diet quality effects.

As shown in Fig. 1, mortality rates of honey bees 72 $h$ post-inoculation significantly differed among the treatment groups (mixed model ANOVA across all treatment groups, $\mathrm{df}=3,54 ; \mathrm{F}=10.03 ; \mathrm{p}<2.34 \mathrm{e}-05)$. The effect of virus treatment (mixed model ANOVA, $\mathrm{df}=1,54 ; \mathrm{F}$ $=24.73 ; \mathrm{p}<7.04 \mathrm{e}-06$ ) and diet treatment (mixed model ANOVA, df $=1,54 ; \mathrm{F}=5.32 ; \mathrm{p}<2.49 \mathrm{e}-02$ ) were significant, but the interaction between the two factors (mixed model ANOVA, df $=1,54 ; \mathrm{F}=4.72 \mathrm{e}-02, \mathrm{p}=8.29 \mathrm{e}-01$ ) was not significant. We compared mortality levels based on pairwise comparisons: For a given diet, honey bees exposed to the virus showed significantly higher mortality rate than honey bees not exposed to the virus. Bees fed rockrose pollen had significantly elevated mortality with virus infection compared to non-inoculated controls (Benjamini-Hochberg, $\mathrm{p}<1.53 \mathrm{e}-03$ ), and bees fed chestnut pollen similarly had significantly elevated mortality with virus infection compared to controls (BenjaminiHochberg, $\mathrm{p}<3.12 \mathrm{e}-03$ ) (Fig. 1).

As shown in Fig. 2, IAPV titers of honey bees $72 \mathrm{~h}$ post-inoculation significantly differed among the treatment groups (mixed model ANOVA across all treatment groups, $\mathrm{df}=3,33 ; \mathrm{F}=6.10 ; \mathrm{p}<2.03 \mathrm{e}-03)$. The effect of virus treatment (mixed model ANOVA, $\mathrm{df}=1,33 ; \mathrm{F}=$
15.04; $\mathrm{p}<4.75 \mathrm{e}-04$ ) was significant, but the diet treatment ( mixed model ANOVA, $\mathrm{df}=1,33 ; \mathrm{F}=2.55 ; \mathrm{p}=1.20 \mathrm{e}-01$ ) and the interaction between the two factors (mixed model ANOVA, $\mathrm{df}=1,33 ; \mathrm{F}=7.02 \mathrm{e}-01, \mathrm{p}=4.08 \mathrm{e}-01$ ) were not significant. We compared IAPV titers based on pairwise comparisons: Bees fed rockrose pollen had significantly elevated IAPV titers with virus infection compared to non-inoculated controls (Benjamini Hochberg, $\mathrm{p}<7.56 \mathrm{e}-$ 03). However, bees fed chestnut pollen did not have significantly elevated IAPV titers with virus infection compared to non-inoculated controls (Benjamini Hochberg, $\mathrm{p}=6.29 \mathrm{e}-02$ ). While many of the non-inoculated treatment groups showed some RT-qPCR amplification (noninoculated average $\mathrm{Ct}=33.92$; inoculated average $\mathrm{Ct}=24.9$ ), and thus have virus titers calculable on a standard curve, these $\mathrm{Ct}$ levels are similar to those deemed uninfected in previous studies [49]. Overall, we interpreted these findings to mean that high quality chestnut pollen could partially reduce high virus titers resulting from the inoculation treatment, whereas low quality rockrose pollen could not (Fig. 2).

\section{Transcriptomic responses to virus infection and diet}

In bees collected $36 \mathrm{~h}$ post treatment, we observed a substantially larger number of differentially expressed genes (DEGs) in our diet main effect $(n=1914)$ than in our virus main effect $(n=43)$ (Additional file 1 : Table S1A and B). There were only four genes that were DEGs in both our diet main effect and our virus main effect (GB48747, GB47214, GB42908, and GB42507). In the diet factor, more DEGs were upregulated in the more-nutritious chestnut group $(n=1033)$ than in the less-nutritious rockrose group $(n=881)$.
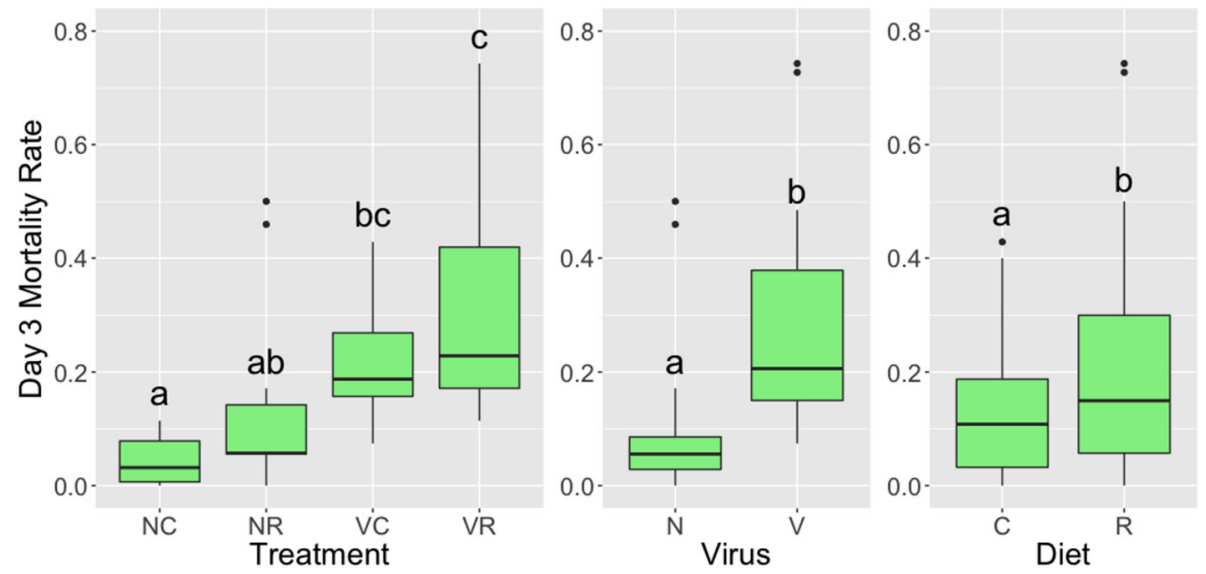

Fig. 1 Mortality rates for the four treatment groups, two virus groups, and two diet groups. Left to right: Mortality rates for the four treatment groups, two virus groups, and two diet groups. " $\mathrm{N}$ " represents non-inoculation, " $\mathrm{V}$ " represents viral inoculation, " $\mathrm{C}$ " represents chestnut pollen, and " $\mathrm{R}$ " represents rockrose pollen. The mortality rate data included 59 samples with 15 replicates per treatment group, except for the "NC" group having 14 replicates. ANOVA values and $p$-values for the statistical tests are listed in the text of the paper. The letters above the bars represent significant differences with a confidence level of $95 \%$ 

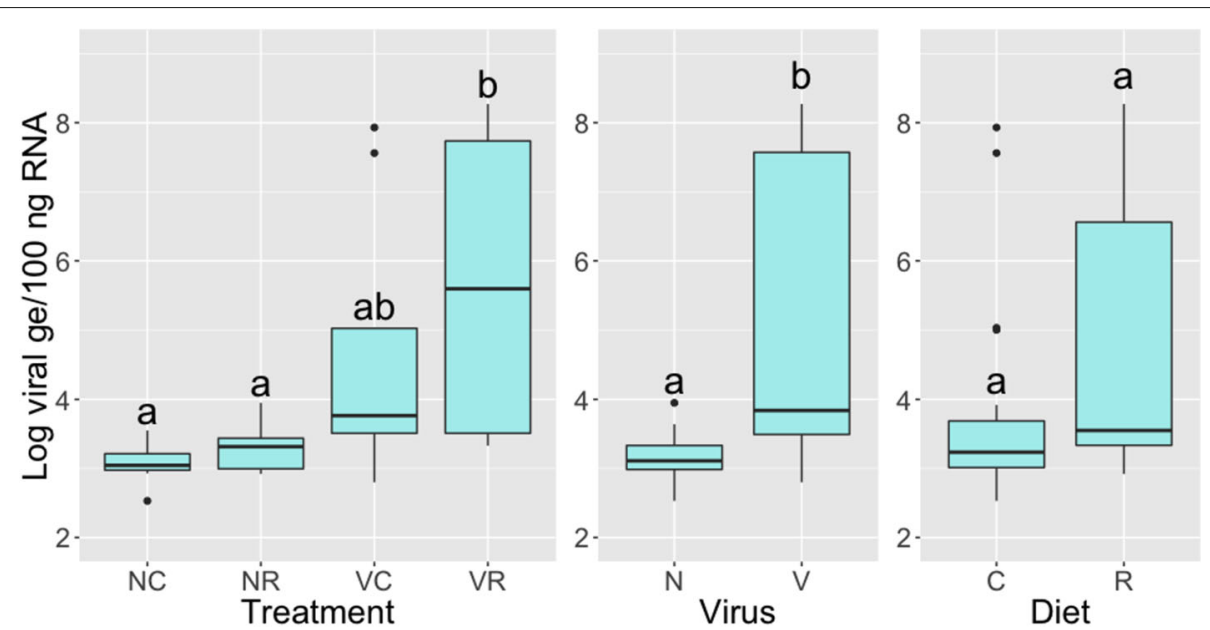

Fig. 2 IAPV titers for the four treatment groups, two virus groups, and two diet groups. Left to right: IAPV titers for the four treatment groups, two virus groups, and two diet groups. " $\mathrm{N}$ " represents non-inoculation, " $\mathrm{V}$ " represents viral inoculation, " $\mathrm{C}$ " represents chestnut pollen, and " $\mathrm{R}$ " represents rockrose pollen. The IAPV titer data included 38 samples with 10 replicates per treatment group, except for the "NR" group having 8 replicates. ANOVA values and $p$-values for the statistical tests are listed in the text of the paper. The letters above the bars represent significant differences with a confidence level of $95 \%$

In the virus factor, there were more virus-upregulated DEGs $(n=38)$ than control-upregulated DEGs $(n=$ 5). While these reported DEG counts are from the DESeq2 package, we saw similar trends for the edgeR and limma package results (Additional file 1: Table S1 and Additional file 18).

We performed GO analysis to statistically assign our DEGs to predefined bins based on their functional characteristics, allowing us to better understand the biological processes of our DEGs. GO analysis of the chestnut-upregulated DEGs revealed the following overrepresented biological functions: Wnt signaling, hippo signaling, and dorso-ventral axis formation, as well as pathways related to circadian rhythm, mRNA surveillance, insulin resistance, inositol phosphate metabolism, FoxO signaling, ECM-receptor interaction, phototransduction, Notch signaling, JaK-STAT signaling, MAPK signaling, and carbon metabolism (Additional file 1: Table S2). These encompassed almost all of the overrepresented biological functions in chestnut-upregulated DEGs conditioned on non-inoculation (i.e. upregulated in the "NC" group compared to the "NR" group; Additional file 1: Table S4) and inoculation (i.e. upregulated in the "VC" group compared to the "VR" group; Additional file 1: Table S6). GO analysis of the rockrose DEGs revealed pathways related to terpenoid backbone biosynthesis, homologous recombination, SNARE interactions in vesicular transport, aminoacyl-tRNA biosynthesis, Fanconi anemia, and pyrimidine metabolism (Additional file 1: Table S3). We note that Fanconi anemia pathways was also the only GO term discovered in rockrose DEGs conditioned on viral inoculation (i.e. upregulated in the "VR" group compared to the "VC" group) (Additional file 1: Table S7). However, Fanconi anemia pathways were not found in rockrose DEGs conditioned on non-inoculation (i.e. upregulated in the "NR" group compared to the "NC" group) (Additional file 1: Table S5).

With so few DEGs $(n=43)$ in our virus main effect comparison, we focused on individual genes and their known functionalities rather than GO overrepresentation (Table 1). Of the 43 virus-related DEGs, only 10 had GO assignments within the DAVID database. These genes had putative roles in the recognition of pathogen-related lipid products and the cleaving of transcripts from viruses, as well as involvement in ubiquitin and proteosome pathways, transcription pathways, apoptotic pathways, oxidoreductase processes, and several more functions (Table 1).

No interaction DEGs were observed between the diet and virus factors of the study, in any of the pipelines (DESeq2, edgeR, and limma).

The number of DEGs across the six treatment pairings between the diet and virus factor ranged from 0 to 955 (Additional file 1: Table S8). Again, diet level appeared to have greater influence on the number of DEGs than the virus level. Across every pair comparing the chestnut and rockrose levels, regardless of the virus level, the number of chestnut-upregulated DEGs was higher than the number of rockrose-upregulated DEGs (Additional file 1: Table S8C-F). Virus-treated bees showed equal to or more upregulated genes relative to controls, under both diet treatments (Additional file 1: Table S8A and B). These trends were observed for all three pipelines used (DESeq2, edgeR, and limma). 
Table 1 Known functions of the mapped subset of 43 DEGs in the virus main effect of our study

\begin{tabular}{|c|c|c|c|c|}
\hline BeeBase ID, NCBI Gene ID & Gene Name & Known functions & Us & Galbraith \\
\hline GB41545, 409187 & MD-2-related lipid-recognition protein-like & $\begin{array}{l}\text { Implicated in lipid recognition, partic- } \\
\text { ularly in the recognition of pathogen } \\
\text { related products }\end{array}$ & $\mathrm{N}$ & - \\
\hline GB50955, 411577 & Protein argonaute-2 & $\begin{array}{l}\text { Interacts with small interfering RNAs to } \\
\text { form RNA-induced silencing complexes } \\
\text { which target and cleave transcripts that } \\
\text { are mostly from viruses and transposons }\end{array}$ & V & V \\
\hline GB48755, 727455 & UBA-like domain-containing protein 2 & $\begin{array}{l}\text { Found in diverse proteins involved in } \\
\text { ubiquitin/proteasome pathways }\end{array}$ & V & V \\
\hline GB47407, 406132 & Histone $\mathrm{H} 4$ & $\begin{array}{l}\text { Capable of affecting transcription, DNA } \\
\text { repair, and DNA replication when post- } \\
\text { transcriptionally modified }\end{array}$ & V & V \\
\hline GB42313, 409923 & Leishmanolysin-like peptidase & $\begin{array}{l}\text { Encodes a protein involved in cell migra- } \\
\text { tion and invasion; implicated in mitotic } \\
\text { progression in D. melanogaster }\end{array}$ & V & V \\
\hline GB50813, 410127 & Rho guanine nucleotide exchange factor 11 & $\begin{array}{l}\text { Implicated in regulation of apoptopic } \\
\text { processes, cell growth, signal transduc- } \\
\text { tion, and transcription }\end{array}$ & V & V \\
\hline GB54503, 411255 & Thioredoxin domain-containing protein & $\begin{array}{l}\text { Serves as a general protein disulphide } \\
\text { oxidoreductase }\end{array}$ & $\mathrm{N}$ & - \\
\hline GB53500, 100576392 & Transcriptional regulator Myc-B & $\begin{array}{l}\text { Regulator gene that codes for a tran- } \\
\text { scription factor }\end{array}$ & V & V \\
\hline GB51305, 551252 & Tropomyosin-like & $\begin{array}{l}\text { Related to protein involved in muscle } \\
\text { contraction }\end{array}$ & $\mathrm{N}$ & $\mathrm{N}$ \\
\hline GB50178, 726905 & Cilia and flagella-associated protein 61-like & $\begin{array}{l}\text { Induces components required for wild- } \\
\text { type motility and stable assembly of } \\
\text { motile cilia }\end{array}$ & V & V \\
\hline
\end{tabular}

Whether the gene was overrepresented in the virus or non-virus group is also indicated for both our study and the Galbraith study. Functionalities were extracted from Flybase, National Center for Biotechnology Information and The European Bioinformatics Institute databases

Additional file 20: Tables S1-9 contain complete DEG lists for all comparisons performed in this study.

\section{Transcriptomic data visualization and comparison to a previous study}

We wished to explore the signal:to:noise ratio between the Galbraith dataset and our dataset. Note that the Galbraith dataset contained three individual bees per treatment group as a single pooled sample, while our dataset contained 16 individual bees per treatment group in 8 RNAseq samples. Basic PCA plots were constructed with the DESeq2 analysis pipeline and showed that the Galbraith dataset may separate the inoculated and non-inoculated honey bees better than our dataset (Additional file 2). Wanting to learn more about the data at the gene level, we continued with new visualization techniques that are available online [72]. For more information about the visualizations used here, please refer to (https://lindsayrutter. github.io/bigPint/articles/plotIntro.html).

We used parallel coordinate lines superimposed onto side-by-side boxplots to visualize the DEGs associated with virus infection in the two studies. The background side-by-side boxplot represents the distribution of all genes in the data (all 15,314 genes in our count table), and each parallel coordinate line represents one DEG. In a parallel coordinate line, connections between samples with positive correlations should be flat, while connections between samples with negative correlations should be crossed. We expect DEGs to show more variability between treatments than between replicates. This means the parallel coordinate lines should be flat between replicates but crossed between treatments. However, overplotting problems would obscure our visualization if we were to plot all DEGs onto the same side-by-side boxplot. Therefore, we graphed clustered subsets of the DEGs (based on hierarchical clustering).

The 1019 DEGs from the Galbraith dataset form relatively clean-looking visual displays, with consistent replicates and differences between treatments. The few inconsistent replicates we observed (such as V.1 from Cluster 1 and V.2 from Cluster 4) were small enough that consistent differences between the treatment groups remained apparent across the samples (Fig. 3). In contrast, we see that the 43 virus-related DEGs from our dataset do not look as clean in their visual displays (Fig. 4). The replicates appear somewhat inconsistent in their estimated expression levels and there is not always such a large (or even consistent) difference between treatment groups. We see 

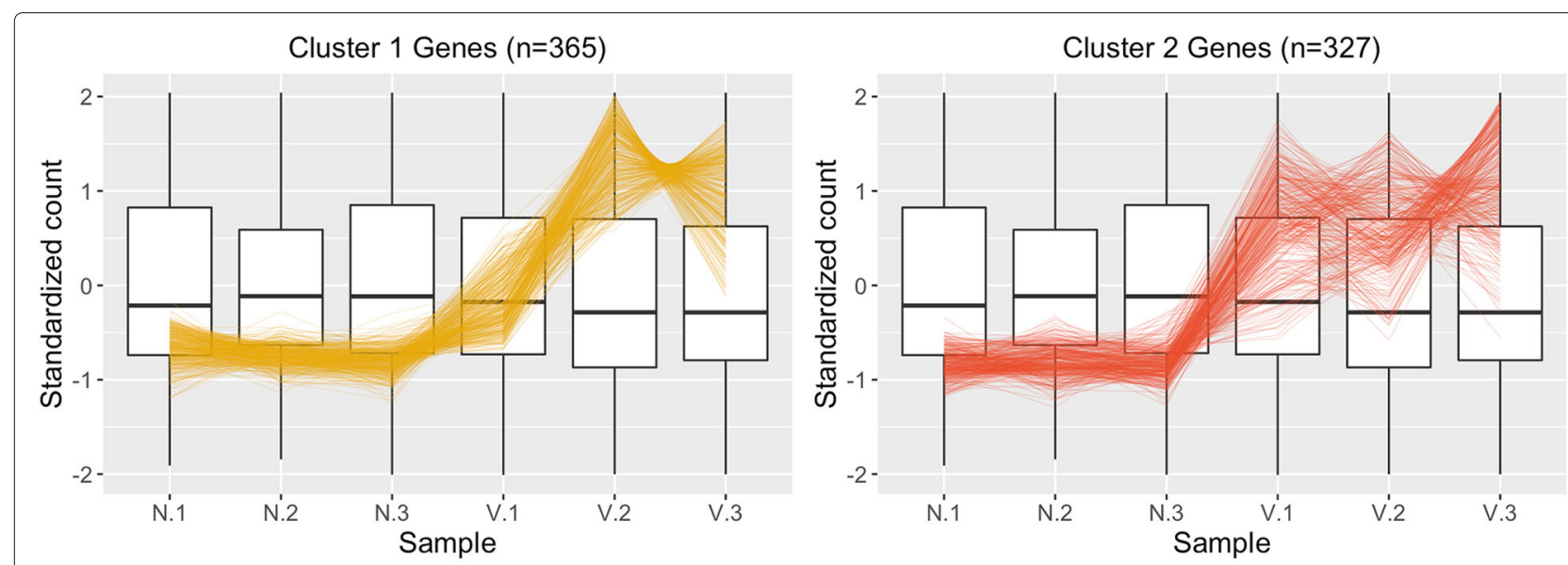

Cluster 3 Genes $(n=224)$
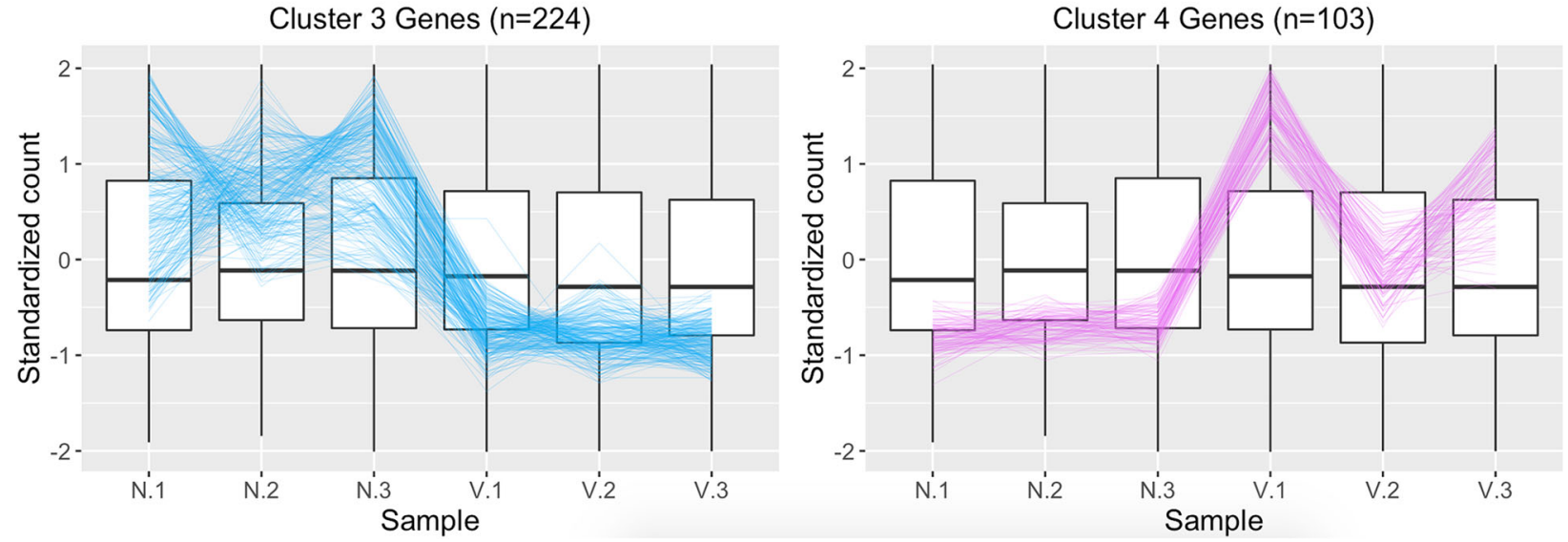

Fig. 3 Parallel coordinate plots of the 1019 virus-related DEGs of the Galbraith data [49]. Parallel coordinate plots of the 1019 DEGs after hierarchical clustering of size four between the virus-infected and control groups of the Galbraith study. " $\mathrm{N}$ " represents non-inoculation, "V" represents viral inoculation. Clusters 1, 2, and 4 seem to represent DEGs that were overexpressed in the virus inoculated group, and Cluster 3 seems to represent DEGs that were overexpressed in the non-inoculated control group. In general, the DEGs appeared as expected, but there is rather noticeable deviation of the first replicate from the virus-treated sample ("V.1") from the other virus-treated replicates in Cluster 1 . We also note a deviation of the second replicate from the virus-treated samples ("V.2") from the other virus-treated replicates in Cluster 4

a similar finding when we also examine a larger subset of 1914 diet-related DEGs from our study (Additional file 3).

We next used repLIcate TREatment ("litre") plots, which we recently developed for our bigPint software package [72]. Litre plots allow users to visualize one DEG onto the Cartesian coordinates of one scatterplot matrix. In the litre plot, each gene in the data is plotted once for every combination of replicates between treatment groups. We use hexagon bins to summarize this massive information. Once the background of hexagons has been drawn to reveal the distribution of all between-treatment sample pair combinations for all genes, the user can superimpose all between-treatment sample pair combinations for one gene of interest.

Additional file 4 shows nine example litre plots for our dataset; each litre plot shows the 144 betweentreatment sample pair combinations for one DEG of interest. Additional files 5 and 6 similarly each show nine example litre plots for the Galbraith dataset; each litre plot shows the nine between-treatment sample pair combinations for one DEG of interest. We see that indeed the virus DEGs from our data (Additional file 4) show less consistent replications and less differences between the treatment groups compared to the virus DEGs from the Galbraith data (Additional files 5 and 6). We also observe that, in the Galbraith dataset, the DEG points in the first cluster show less tight cluster patterns than the DEG points in the second cluster (Additional files 5 and 6), an observation we saw previously in the parallel coordinate plots (Fig. 3).

Finally, we used scatterplot matrices from the bigPint software to further assess the DEGs [72]. A scatterplot matrix is another effective multivariate visualization tool that plots read count distributions across all genes and samples. Specifically, it represents every gene in the dataset as a black point in each scatterplot. DEGs can 


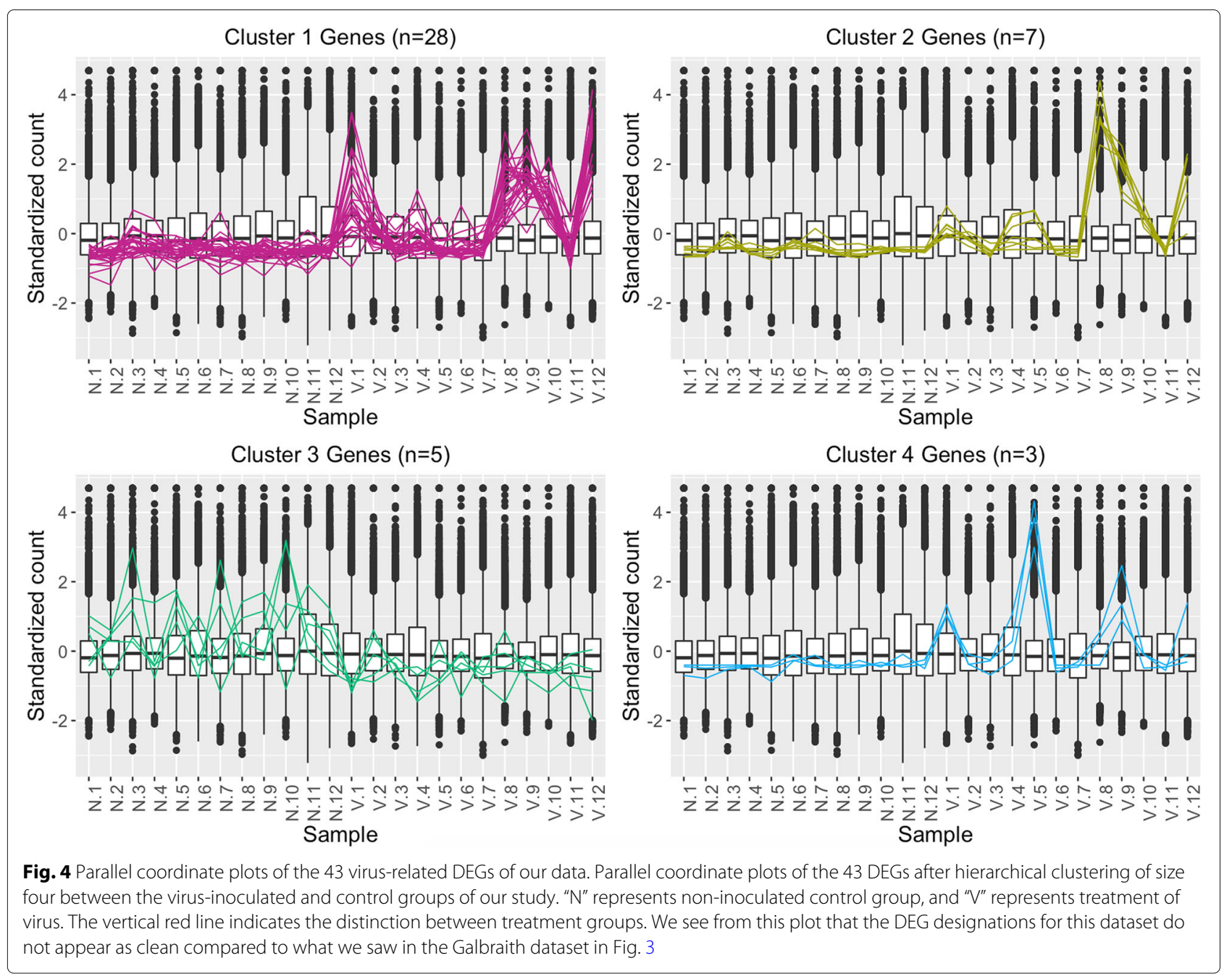

be superimposed as colored points to assess their patterns against the full dataset. We expect DEGs to mostly fall along the $x=y$ line in replicate scatterplots (denoting replicate consistency) but deviate from the $x=y$ line in treatment scatterplots (denoting significant treatment changes). The $x=y$ line is shown in red in our plots.

We created standardized scatterplot matrices for each of the four clusters (from Fig. 3) of the Galbraith data (Additional files 7, 8, 9, and 10). We also created standardized scatterplot matrices for our data. However, as our dataset contained 24 samples, we would need to include 276 scatterplots in our matrix, which would be too numerous to allow for efficient visual assessment of the data. As a result, we created four scatterplot matrices of our data, each with subsets of 6 samples to be more comparable to the Galbraith data (Additional files 11, 12, 13, and 14). Specifically, we arbitrarily subsetted the samples so each one was represented once in each of these four files (i.e. Additional file 11 shows samples 1-3; Additional file 12 shows samples 4-6; Additional file 13 shows samples 7-9; and Additional file 14 shows samples 10-12). We can again confirm through these plots that the DEGs from the Galbraith data appeared more as expected: They deviated more from the $x=y$ line in the treatment scatterplots while staying close to the $x=y$ line in replicate scatterplots.

Despite the virus-related DEGs $(n=1019)$ from the Galbraith dataset displaying the expected patterns more than those from our dataset $(n=43)$, there was significant overlap ( $p$-value $<2.2 \mathrm{e}-16$ ) in the DEGs between the two studies, with 26/38 (68\%) of virus-upregulated DEGs from our study also showing virus-upregulated response in the Galbraith study (Fig. 6).

\section{Tolerance versus resistance}

Using the contrasts specified in Table 2, we discovered 122 "tolerance" candidate DEGs and 125 "resistance" candidate DEGs. Within our 122 "tolerance" gene ontologies, we found functions related to metabolism (such as carbohydrate metabolism, fructose metabolism, and chitin metabolism). However, we also discovered gene 
Table 2 Contrasts in our study for assessing GO and pathways analysis

\begin{tabular}{|c|c|c|c|}
\hline Contrast & DEGs & Interpretation & Results \\
\hline V (all) vs N (all) & 43 & $\begin{array}{l}\text { Genes that change expression due to } \\
\text { virus effect regardless of diet status in } \\
\text { bees }\end{array}$ & Table 1 \\
\hline NC vs NR & 941 & $\begin{array}{l}\text { Genes that change expression due to } \\
\text { diet effect in non-inoculated bees }\end{array}$ & $\begin{array}{l}\text { Additional file 1: Table S4 } \\
\text { and } 5\end{array}$ \\
\hline VC VS VR & 376 & $\begin{array}{l}\text { Genes that change expression due to } \\
\text { diet effect in inoculated bees }\end{array}$ & $\begin{array}{l}\text { Additional file 1: Table S6 } \\
\text { and } 7\end{array}$ \\
\hline VC upregulated in VC vs VR, and NC upregulated in NC vs NR & 122 & $\begin{array}{l}\text { "Tolerance" genes that turn on by good } \\
\text { diet regardless of virus infection status in } \\
\text { bees }\end{array}$ & Fig. $5 a$ \\
\hline VC upregulated in VC vs VR, but NC not upregulated in NC vs NR & 125 & $\begin{array}{l}\text { "Resistance" genes that turn on by good } \\
\text { diet only in inoculated bees }\end{array}$ & Fig. $5 b$ \\
\hline
\end{tabular}

ontologies related to RNA polymerase II transcription, immune response, and regulation of response to reactive oxygen species (Fig. 5a). Within our 125 "resistance" gene ontologies, we found functions related to metabolism (such as carbohydrate metabolism, chitin metabolism, oligosaccharide biosynthesis, and general metabolism) (Fig. 5b).

To visually explore gene expression patterns related to tolerance and resistance, we used hierarchical clustering to separate candidate DEGs into common patterns, and then visualized these clusters using parallel coordinate lines superimposed onto side-by-side boxplots. To reduce overplotting of parallel coordinate lines, we again used hierarchical clustering techniques to separate DEGs into common patterns. Perhaps unsurprisingly, we still see a substantial amount of noise (inconsistency between replicates) in our resulting candidate DEGs (Additional files 15 and 16). However, the broad patterns we expect to see still emerge: For example, based on the contrasts we created to obtain the "tolerance" candidate DEGs, we expect them to display larger count values in the "NC" group compared to the "NR" group and larger count values in the "VC" group compared to the "VR" group. Indeed, we see this pattern in the associated parallel coordinate plots (Additional file 15). Likewise, based on the contrasts we created to obtain the "resistance" candidate DEGs, we still expect them to display larger count values in the "VC" group compared to the "VR" group, but we no longer expect to see a difference between the "NC" and "NR" groups. We do generally see these expected patterns in the associated parallel coordinate plots: While there are large outliers in the "NC" group, the "NR" replicates are no longer typically below a standardized count of zero (Additional file 16). The genes in Cluster 3 follow the expected pattern the most distinctively (Additional file 16).

\section{Post hoc analysis}

To better understand sources of transcriptomic noise, we explored whether pathogen response measurements (virus titers and mortality), which varied widely across samples, were correlated with observed patterns in gene expression.

The R-squared values between gene read counts and pathogen response measurements were generally low (R-squared <0.1) across our dataset (Additional file 1: Table S9). We further explored whether clusters of DEGs showed higher correlations with pathogen response measurements than non-DEGs (the latter serving as a control, where we do not expect a correlation). A Kruskal-Wallis test was used to determine if R-squared distributions of DEG clusters significantly differed from those in the rest of the data. The $p$-values and Bonferroni correction values for each of the 36 tests (as described in the methods section) is provided in Additional file 1: Table S9. Distribution of the R-squared values for DEG cluster read counts and pathogen response metrics is provided in Additional file 19. An overall trend emerges to suggest that DEGs may have significantly larger correlation with the pathogen response measurements compared to non-DEGs.

\section{Discussion}

Challenges to honey bee health are a growing concern, in particular the combined, interactive effects of nutritional stress and pathogens [12]. In this study, we used RNA-sequencing to probe mechanisms underlying honey bee responses to two effects, diet quality and infection with the prominent virus of concern, IAPV. In general, we found a major nutritional transcriptomic response, with nearly 2000 transcripts changing in response to diet quality (rockrose/poor diet versus chestnut/good diet). The majority of these genes were upregulated in response to high quality diet, and these genes were over-represented for functions such as nutrient signaling metabolism (insulin resistance), immune response (Notch signaling and JaK-STAT pathways), and carbon metabolism (Additional file 1: Table S2). These data suggest high quality nutrition may allow bees to alter their 

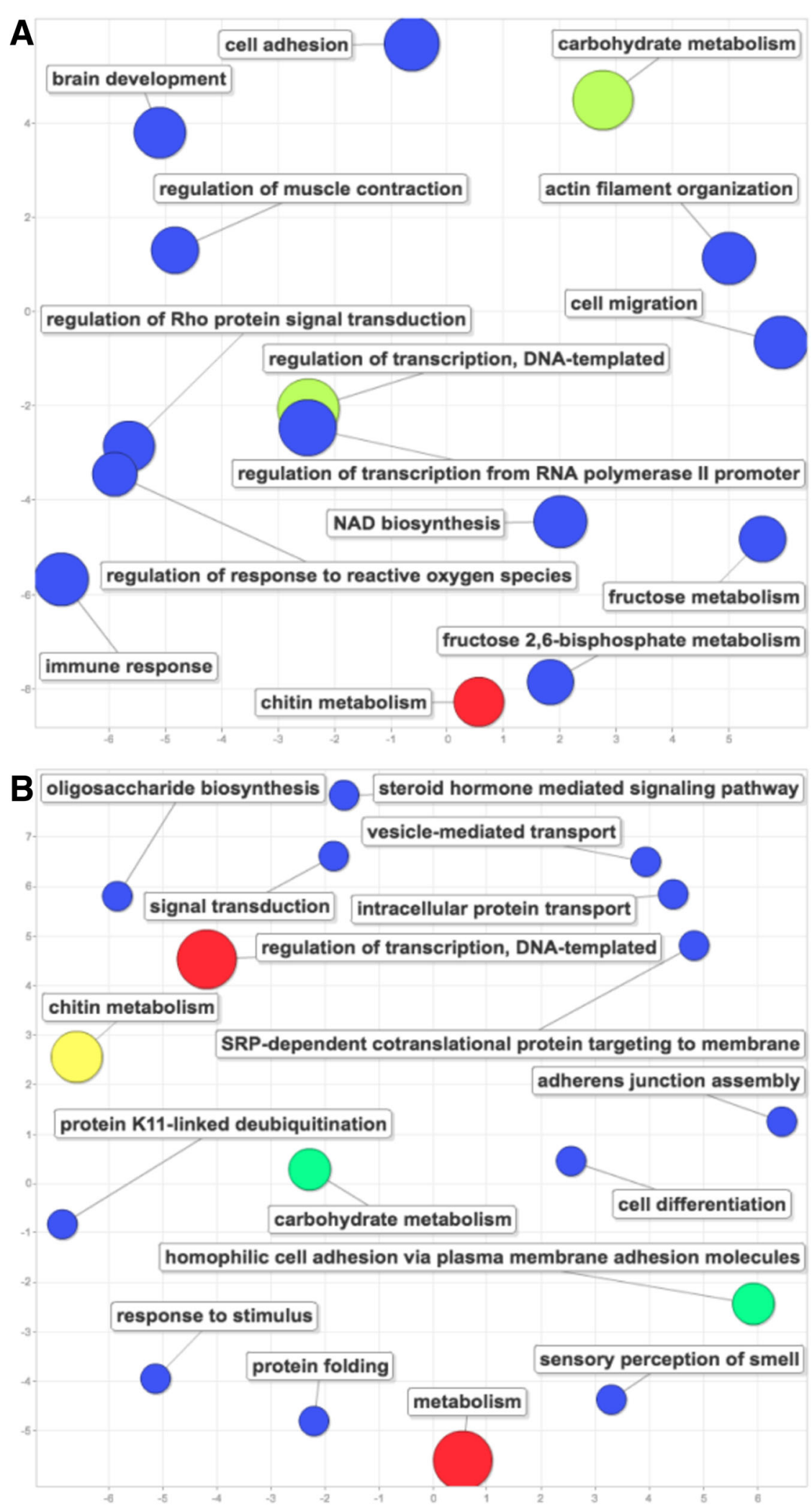

Fig. 5 Gene ontology results for the 122 "tolerance" and 125 "resistance" DEG candidates in our data. GO analysis results for the 122 DEGs related to our "tolerance" hypothesis (A) and for the 125 DEGs related to our "resistance" hypothesis (B). The color and size of the circles both represent the number of genes in that ontology. The $x$-axis and $y$-axis are organized by SimRel, a semantic similarity metric [106] 
metabolism, favoring investment of energy into immune responses.

One of the few studies that has investigated transcriptomic response to nutrition in honey bees similarly found that pollen upregulates genes related to macromolecule metabolism, insulin pathways, and TOR pathways [48]. Diet effects on transcriptomics have been more extensively studied in the insect model Drosophila. One recent transcriptomic study in Drosophila melanogaster reported an overexpression of genes related to immunity, metabolism, and hemocyanin in a high-fat diet and overexpression of genes related to cell cycle activity, DNA binding and transcription, and CHK kinase-like protein activity in a high-sugar diet [54]. This same study also discovered an upregulation of genes related to peptide and carbohydrate processing in both high-fat and high-sugar diets, a finding the authors attributed to a general increase in caloric intake. Another recent study investigated the transcriptomic effects of diets high in protein relative to sugar, diets high in sugar relative to protein, and diets with equal amounts of protein and sugar [55]. Drosophila mojavensis and Drosophila arizonae showed substantial differential expression between the dietary conditions: genes involved in carbohydrate and lipid metabolism were upregulated in response to high sugar low protein diets and genes involved in juvenile hormone $(\mathrm{JH})$ and ecdysone were upregulated in response to low sugar high protein diets. Interestingly, prior studies have suggested that $\mathrm{JH}$ regulates body size by controlling ecdysone production, which modifies insulin signaling [56]. As we saw in our study, these studies generally suggest that diet differences may relate to gene expression changes in metabolism and immune responses in honey bees.

While some insect systems have shown relatively low transcriptional responses to dicistrovirus infection [93, 94], previous work on honey bees has revealed many hundreds of DEGs [49]. Discrepancies between datasets may be due to noise and complexity of the honey bee microbiome. The transcriptomic response to virus infection in our experiment was fairly limited. We found only 43 differentially expressed transcripts, some with known immune functions such as an MD-2 lipid recognition protein that is particularly implicated in the recognition of pathogen products and argonaute-2, a protein that plays a central role in RNA silencing (Table 1). We also found genes related to transcriptional regulation, including Histone $\mathrm{H} 4$, Rho guanine nucleotide exchange factor 11 , and transcriptional regular $\mathrm{Myc}-\mathrm{B}$, which is a regular gene that codes for a transcription factor. We additionally found Tropomyosin-like, a gene involved in muscle contraction. The small number of DEGs in this study may be partly explained by the large amount of noise in the data (Fig. 4 and Additional files 2B, 4, 11, 12, 13, and 14) and baseline viral titers observed in our control bees (Fig. 2).
There have been numerous studies on the transcriptomic effects of virus infection in model organisms like fruit flies and mosquitoes that can provide a useful framework for interpreting virus responses in honey bees. These studies have showed that RNA silencing is a major antiviral strategy, along with transcriptional pausing, Toll pathways, IMD pathways, JAK/STAT pathways, and Toll7 -autophagy pathways $[60,61]$. Recent transcriptomic studies in honey bees have shown similar hallmarks of these same antiviral defense mechanisms, including RNA silencing, Toll pathways, IMD pathways, JAK/STAT pathways, autophagy, and endocytosis [62]. It is important to note that general immune responses to viral infection in insects might be an indirect result of cellular damage [61]. In fact, every virus-host interaction has its own particularities derived from the diverse methods of replication and infection cycle evolved by different viruses. An intricate set of pro- and anti-virus host factors such as ribosomal proteins and autophagy pathways are involved, but the response depends on the virus species, as has been elucidated in Drosophila $[60,61]$. In addition, a non-sequence-specific antiviral response mediated by unspecific dsRNA pathway was discovered in honey bees $[50,95]$. In the case of dicistroviruses, few works have studied the impact of IAPV infection at transcriptional level. Chen et al. 2014 analyzed responses to IAPV infection in larvae and workers using microarrays [51]. Many of the DEGs found were involved in immune response and energy-related metabolism, particularly in adults but not in brood. The authors propose this observed difference could be connected to latent infections in larvae (where host immunity is not perturbed) versus acute infections in adulthood (induced by stressors faced during development) [51]. IAPV acute infection also alters the DNA methylation pattern of numerous genes that do not overlap the genes that are up- or down-regulated at the transcriptional level [49]. These works reiterate the conclusion that viruses trigger particular antiviral mechanisms by different means and depending on several factors. The honey bee antiviral pathways induced by specific viruses were recently reviewed [62]; it is noteworthy that many honey bee factors discovered by transcriptomics need further characterization to uncover their role in controlling (or promoting) viral infection in honey bees.

Given the noisy nature of our data, and our desire to home in on genes with real expression differences, we compared our data to the Galbraith study [49], which also examined bees response to IAPV infection. In contrast to our study, Galbraith et al. identified a large number of virus responsive transcripts, and generally had less noise in their data (Fig. 3 and Additional files 2A, 5, 6, 7, 8, 9, and 10). To identify the most consistent virus-responsive genes from our study, we looked for overlap in the DEGs associated with virus infection on both experiments. We 
found a large, statistically significant ( $p$-value $<2.2 \mathrm{e}-16$ ) overlap, with $26 / 38$ (68\%) of virus-responsive DEGs from our study also showing response to virus infection in Galbraith et al. (Fig. 6). This result gives us confidence that, although noisy, we were able to uncover reliable, replicable gene expression responses to virus infection with our data.

Data visualization is a useful method to identify noise and robustness in RNA-sequencing data [79]. In this study, we used extensive data visualization to improve the interpretation of our RNA-sequencing results. For example, the DESeq2 package comes with certain visualization options that are popular in RNA-sequencing analysis. One of these visualization is the principal component analysis (PCA) plot, which allows users to visualize the similarity between samples within a dataset. We could determine from this plot that indeed the Galbraith data may show more similarity between its replicates and differences between its treatments compared to our data (Additional file 2). However, the PCA plot only shows us information at the sample level. We wanted to investigate how these differences in the signal:to:noise ratios of the datasets would affect the structure of any resulting DEGs. As a result, we also used three plotting techniques from the bigPint package: We investigated the 1019 virus-related DEGs from the Galbraith dataset and the 43 virus-related DEGs from our dataset using parallel coordinate lines, scatterplot matrices, and litre plots. To prevent overplotting issues in our graphics, we used a hierarchical clustering technique for the parallel coordinate lines to separate the set of DEGs into smaller groups. We also needed to examine four subsets of samples from our dataset to make effective use of the scatterplot matrices. After these tailorizations, we determined that the same patterns we saw in the PCA plots regarding the entire dataset extended down the pipeline analysis into the DEG calls: Even the DEGs from the Galbraith dataset showed more similarity between their replicates and differences between their treatments compared to those from our data. However, the 365 DEGs from the Galbraith data in Cluster 1 of Fig. 3 showed an inconsistent first replicate in the treatment group ("V.1"), which was something we observed in the PCA plot. This indicates that this feature also extended down the analysis pipeline into DEG calls. Despite the differences in signal between these two datasets, there was substantial overlap in the resulting DEGs. We believe these visualization applications can be useful for future researchers analyzing RNAsequencing data to quickly and effectively ensure that the DEG calls look reliable or at least overlap with DEG calls from similar studies that look reliable. We also expect this type of visualization exploration can be especially crucial when studying wild populations with high levels of genetic and environmental variation between replicates and/or when using experiments that may lack rigid design control.

One of the goals of this study was to use our RNA-sequencing data to assess whether transcriptomic responses to diet quality and virus infection provide insight into whether high quality diet can buffer bees from pathogen stress via mechanisms of "resistance" or "tolerance". Recent evidence has suggested that overall immunity is determined by more than just "resistance" (the reduction of pathogen fitness within the host by mechanisms of avoidance and control) [96]. Instead, overall immunity is related to "resistance" in conjunction with "tolerance" (the reduction of adverse effects and disease resulting from pathogens by mechanisms of healing) $[96,97]$. Immune-mediated resistance and diet-driven tolerance mechanisms are costly and may compete with each other [97, 98]. Data and models have suggested that selection can favor an optimum combination of
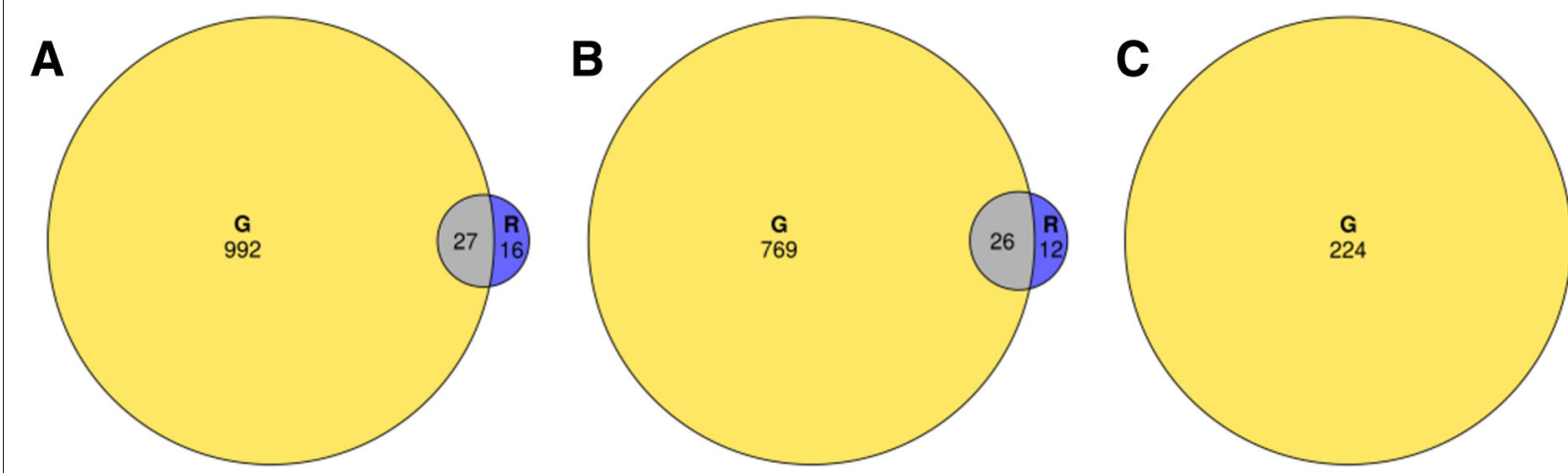

Fig. 6 Venn diagrams comparing the virus-related DEG overlaps between our dataset and the Galbraith dataset. Venn diagrams comparing the virus-related DEG overlaps between the Galbraith study (labeled as " $G$ ") and our study (labeled as " $R$ "). From left to right: Total virus-related DEGs (subplot A), virus-upregulated DEGs (subplot B), control-upregulated DEGs (subplot C). Both the total virus-related and virus-upregulated DEGs showed significant overlap between the studies ( $p$-value $<2.2 \mathrm{e}-16$ ) as per Fisher's Exact Test for Count Data. There was one gene that was virus-upregulated in the Galbraith study but control-upregulated in our study 
both resistance and tolerance [99-102]. We attempted to address this topic through specific gene expression contrasts (Table 2), accompanied by GO analysis of the associated gene lists. We found an approximately equal number of resistance $(n=125)$ and tolerance $(n=122)$ related candidate DEGs, suggesting both processes may be playing significant roles in dietary buffering from pathogen induced mortality. Resistance candidate DEGs had functions related to several forms of metabolism (chitin and carbohydrate), regulation of transcription, and cell adhesion (Fig. 5b). Tolerance candidate DEGs had functions related to carbohydrate metabolism and chitin metabolism; however, they also showed functions related to immune response, including RNA polymerase II transcription (Fig. 5a). Previous studies have shown that transcriptional pausing of RNA polymerase II may be an innate immune response in D. melanogaster that allows for a more rapid response by increasing the accessibility of promoter regions of virally induced genes [103]. These possible immunological defense mechanisms within our "tolerance" candidate DEGs and metabolic processes within our "resistance" candidate DEGs may provide additional evidence of feedbacks between diet and disease in honey bees [12]. Thus, our study uses transcriptome data to generate lists of candidate genes that can be the focus of future investigations to better experimentally test putative roles of tolerance and resistance genes in this system.

There were several limitations in this study that could be improved upon in future studies. For instance, our comparison between the Galbraith data (single-drone colonies) and our data (naturally-mated colonies) was limited by numerous extraneous variables between these studies. In addition to different molecular pipelines and bioinformatic preprocessing pipelines used between these studies, the Galbraith study focused on worker honey bees that were fed sugar and artificial pollen diets, whereas we used whole bodies and categorized only into inoculated vs. non-inoculated groups; noise may have been introduced through different responses in asymptomatic bees. Also, Galbraith's bees were sampled at $24 \mathrm{~h}$ while ours were sampled at $36 \mathrm{~h}$. Furthermore, the Galbraith data used eviscerated abdomens with attached fat bodies and observations to determine behaviorally symptomatic bees whereas we used whole bodies and categorized only into inoculated vs. non-inoculated groups. There are also differences in the hours post inoculation and possible differences in the inoculation amount between the studies. Further differences between the studies can be found in their corresponding published methods sections $[11,49]$. The different factors between these two studies may be critical because particular antiviral factors in honey bees are linked to specific viruses, specific developmental stages, the analyzed tissue, the route of inoculation, and the time (post-inoculation) during which the study was performed. This was clearly demonstrated when comparing honey bee responses to two related iflaviruses with very different infection dynamics, SBV vs. DWV [52]. Authors observed differences in induction of defensin and hymenoptaecin immune-related genes, and suggested the results reflect adaptations to the different routes of transmission [52].

Moreover, our comparative visualization assessment between these two datasets was also somewhat limited because the virus effect in the Galbraith study used three replicates for each level, whereas the virus effect in our study used twelve replicates for each level that were actually further subdivided into six replicates for each diet level. Hence the apparent reduction in noise observed in the Galbraith data compared to our data in the PCA plots, parallel coordinate plots, scatterplot matrices, and litre plots may be an inadvertent product of the smaller number of replicates used and the lack of a secondary treatment group rather than solely the reduction in genetic variability through the single-drone colony design itself. With this in mind, while our current efforts may be a starting point, future studies can shed more light on signal:to:noise and differential expression differences between naturally-mated colony designs and single-drone colony designs by controlling for extraneous factors more strictly than what we were able to do in the current line of work.

In addition, this study used a whole body RNAsequencing approach. In future related studies, it may be informative to use tissue-specific methods. Previous work has shown that even though IAPV replication occurs in all honey bee tissues, it localizes more in gut and nerve tissues and in the hypopharyngeal glands. Likewise, the highest IAPV titers have been observed in gut tissues [41]. Recent evidence suggests that RNA-sequencing of composite structures (rather than specific tissues) in honey bees leads to false negatives, implying that genes strongly differentially expressed in particular structures may not reach significance within the composite structure [104]. These studies have also found that within a composite extraction, structures therein may contain opposite patterns of differential expression. If we were to repeat this same experimental design at a more refined tissue level, we would likely provide more detailed answers to our original transcriptomic questions. Another future direction related to this work would be to integrate multiple omics datasets to investigate monofloral diet quality and IAPV infection in honey bees. Indeed, previous studies in honey bees have found that multiple omics datasets do not always align in a clear-cut manner, and hence may broaden our understanding of the molecular mechanisms being explored [49]. 


\section{Conclusions}

To the best of our knowledge, there are few to no studies investigating honey bee gene expression specifically related to monofloral diets, and few to no studies examining honey bee gene expression related to the combined effects of diet in any general sense and viral inoculation in any general sense. It also remains unknown whether the protective effects of good diet in honey bees is due to direct effects on immune function (resistance) or indirect effects of energy availability on vigor and health (tolerance). We attempted to address these unresolved areas by conducting a two-factor RNA-sequencing study that examined how monofloral diets and IAPV inoculation influence gene expression patterns in honey bees. Overall, our data suggest complex transcriptomic responses to multiple stressors in honey bees. Diet has the capacity for large and profound effects on gene expression and may set up the potential for both resistance and tolerance to viral infection, adding to previous evidence of possible feedbacks between diet and disease in honey bees [12].

Moreover, this study also demonstrated the benefits of using data visualizations and multiple datasets to address inherently messy biological data. For instance, by verifying the substantial overlap in our DEG lists to those obtained in another study that addressed a similar question using specimens with less genetic variability, we were able to place much higher confidence in the differential gene expression results from our otherwise noisy data. We also suggested that comparing results derived from multiple studies varying in level of genetic and environmental variability may allow researchers to identify transcriptomic patterns that are concurrently more realistic and less noisy. Altogether, we hope our results underline the merits of using data visualization techniques and multiple datasets to understand and interpret RNA-sequencing datasets.

\section{Additional files}

\section{Additional file 1: Supplementary tables.}

In all tables, " $C$ " represents chestnut diet, " $\mathrm{R}$ " represents rockrose diet, " $\mathrm{N}$ " represents control non-inoculated, and "V" represents virus-inoculated.

Table 1: Number of DEGs across three analysis pipelines for the (A) diet main effect in our study, (B) virus main effect in our study, and (C) virus main effect in the Galbraith study. Table 2: Pathways related to the 1,033 DEGs upregulated in the chestnut treatment from the diet main effect. Table 3: Pathways related to the 881 DEGs upregulated in the rockrose treatment from the diet main effect. Table 4: $\mathrm{GO}$ analysis results for the 601 DEGs upregulated in the NC treatment from the NC versus NR treatment pair analysis. These DEGs represent genes upregulated in noninoculated honey bees given high quality chestnut pollen versus low quality rockrose pollen. Table 5: GO analysis results for the 340 DEGs upregulated in the NR treatment from the NC versus NR treatment pair analysis. These DEGs represent genes upregulated in noninoculated honey bees given low quality rockrose pollen versus high quality chestnut pollen. Table 6: $G$ analysis results for the 247 DEGs upregulated in the VC treatment from the VC versus VR treatment pair analysis. These DEGs represent genes upregulated in inoculated honey bees given high quality chestnut pollen versus low quality rockrose pollen. Table 7: $\mathrm{GO}$ analysis results for the 129 DEGs upregulated in the VR treatment from the VC versus VR treatment pair analysis. These DEGs represent genes upregulated in inoculated honey bees given low quality rockrose pollen versus high quality chestnut pollen. Table 8: Number of DEGs across three analysis pipelines for all six treatment pair combinati ons between the diet and virus factor. Table 9: Kruskal-Wallis p-value and Bonferroni corrections for the 36 combinations of DEG lists, pathogen response metrics, and cluster number. (XLS $28 \mathrm{~kb})$.

Additional file 2: PCA plots for the Galbraith dataset and for our dataset. PCA plots for the Galbraith dataset (A) and for our dataset (B). "V" represents virus-inoculated, and " $\mathrm{N}$ " represents control non-inoculated.

The $x$-axis represents the principal component with the most variation and the $y$-axis represents the principal component with the second-most variation. (PNG $250 \mathrm{~kb}$ )

Additional file 3: Parallel coordinate lines of the diet-related DEGs of our dataset.

Parallel coordinate plots of the 1914 DEGs after hierarchical clustering of size six between the chestnut and rockrose groups of our study. Here " $\mathrm{C}$ " represents chestnut samples, and " $\mathrm{R}$ " represents rockrose samples. The vertical red line indicates the distinction between treatment groups. We see from this plot that the DEG designations for this dataset do not appear as clean compared to what we saw in the Galbraith dataset in Fig. 3. (PNG $2031 \mathrm{~kb})$

Additional file 4: Example litre plots from the virus-related DEGs of our dataset.

Example litre plots of the nine DEGs with the lowest FDR values from the 43 virus-related DEGs of our dataset. " $\mathrm{N}$ " represents non-inoculated control samples and "V" represents virus-treated samples. Most of the magenta points (representing the 144 combinations of samples between treatment groups for a given DEG) do not reflect the expected pattern as clearly compared to what we saw in the litre plots of the Galbraith data. They are not as clustered together (representing replicate inconsistency) and they sometimes cross the $x=y$ line (representing lack of difference between treatment groups). This finding reflects what we saw in the messy looking parallel coordinate lines of Fig. 4. (PNG $1160 \mathrm{~kb}$ )

Additional file 5: Example litre plots of DEGs from Cluster 1 of the Galbraith dataset.

Example litre plots of the nine DEGs with the lowest FDR values from the 365 DEGs in Cluster 1 (originally shown in Fig. 3) of the Galbraith dataset. " $\mathrm{N}$ " represents non-inoculated control samples and "V" represents virus-treated samples. Most of the light orange points (representing the nine combinations of samples between treatment groups for a given $D E G$ ) deviate from the $x=y$ line in a tight bundle as expected. (PNG $964 \mathrm{~kb}$ )

Additional file 6: Example litre plots of DEGs from Cluster 2 of the Galbraith dataset.

Example litre plots of the nine DEGs with the lowest FDR values from the 327 DEGs in Cluster 2 (originally shown in Fig. 3) of the Galbraith dataset. " $\mathrm{N}$ " represents non-inoculated control samples and " $\mathrm{V}$ " represents virus-treated samples. Most of the dark orange points (representing the nine combinations of samples between treatment groups for a given $D E G$ ) deviate from the $x=y$ line in a compact clump as expected. However, they are not as tightly bunched together compared to what we saw in the example litre plots of Cluster 1 (shown in Additional file 5). As a result, what we see in these litre plots reflects what we saw in the parallel coordinate lines of Fig. 3: The replicate consistency in the Cluster 1 DEGs is not as clean as that in the Cluster 2 DEGs, but is still relatively clean. (PNG $1018 \mathrm{~kb}$ )

Additional file 7: Scatterplot matrix of DEGs from Cluster 1 of the Galbraith dataset.

The 365 DEGs from the first cluster of the Galbraith dataset (originally shown in Fig. 3) superimposed as light orange dots onto all genes as black dots in the form of a scatterplot matrix. The data has been standardized. " $\mathrm{N}$ " represents non-inoculated control samples and "V" represents virus-treated samples. We confirm that the DEGs mostly follow the expected structure, with their placement deviating from the $x=y$ line in the treatment scatterplots, but adhering to the $x=y$ line in the replicate scatterplots. However, we do see that sample "V.1" may be somewhat inconsistent in these DEGs, as its presence in the replicate scatterplots 
shows DEGs deviating from the $x=y$ line more than expected and its presence in the treatment scatterplots shows DEGs adhering to the $x=y$ line more than expected. This inconsistent sample was something we observed in Fig. 3. (PNG 562 kb)

Additional file 8: Scatterplot matrix of DEGs from Cluster 2 of the Galbraith dataset.

The 327 DEGs from the second cluster of the Galbraith dataset (originally shown in Fig. 3) superimposed as dark orange dots onto all genes as black dots in the form of a scatterplot matrix. The data has been standardized. " $\mathrm{N}$ " represents non-inoculated control samples and " $\mathrm{V}$ " represents virus-treated samples. We confirm that the DEGs mostly follow the expected structure, with their placement deviating from the $x=y$ line in the treatment scatterplots, but adhering to the $x=y$ line in the replicate scatterplots. (PNG $589 \mathrm{~kb}$ )

Additional file 9: Scatterplot matrix of DEGs from Cluster 3 of the Galbraith dataset.

The 224 DEGs from the third cluster of the Galbraith dataset (originally shown in Fig. 3) superimposed as turquoise dots onto all genes as black dots in the form of a scatterplot matrix. The data has been standardized. " $\mathrm{N}$ " represents non-inoculated control samples and "V" represents virus-treated samples. We confirm that the DEGs mostly follow the expected structure, with their placement deviating from the $x=y$ line in the treatment scatterplots, but adhering to the $x=y$ line in the replicate scatterplots. (PNG $618 \mathrm{~kb}$ )

Additional file 10: Scatterplot matrix of DEGs from Cluster 4 of the Galbraith dataset.

The 103 DEGs from the fourth cluster of the Galbraith dataset (originally shown in Fig. 3) superimposed as pink dots onto all genes as black dots in the form of a scatterplot matrix. The data has been standardized. " $\mathrm{N}$ " represents non-inoculated control samples and "V" represents virus-treated samples. We confirm that the DEGs mostly follow the expected structure, with their placement deviating from the $x=y$ line in the treatment scatterplots, but adhering to the $x=y$ line in the replicate scatterplots. We also see that the second replicate from the virus-treated sample ("V.2") may be somewhat inconsistent in these DEGs, as its presence in the replicate scatterplots results in the DEGs unexpectedly deviating from the $x=y$ line and its presence in the treatment scatterplots results in the DEGs unexpectedly adhering to the $x=y$ line. This inconsistent sample was something we observed in Fig. 3. (PNG $560 \mathrm{~kb}$ )

Additional file 11: Scatterplot matrix of virus-related DEGs from our dataset, showing only replicates 1,2 , and 3 .

The 43 virus-related DEGs from our dataset superimposed as magenta dots onto all genes in the form of a scatterplot matrix. Only replicates 1,2, and 3 are shown from both treatment groups. The data has been standardized. " $\mathrm{N}$ " represents non-inoculated control samples and " $\mathrm{V}$ " represents virus-treated samples. We see that, compared to the scatterplot matrices from certain clusters of the Galbraith data, the 43 DEGs from this subset of six samples from our data do not paint as clear of a picture, sometimes unexpectedly deviating from the $x=y$ line in the replicate plots and sometimes unexpectedly adhering to the $x=y$ line in the treatment plots. (PNG $584 \mathrm{~kb}$ )

Additional file 12: Scatterplot matrix of virus-related DEGs from our dataset, showing only replicates 4,5 , and 6 .

The 43 virus-related DEGs from our dataset superimposed as magenta dots onto all genes in the form of a scatterplot matrix. Only replicates 4, 5, and 6 are shown from both treatment groups. The data has been standardized. " $\mathrm{N}$ " represents non-inoculated control samples and " $\mathrm{V}$ " represents virustreated samples. We see that, compared to the scatterplot matrices from certain clusters of the Galbraith data, the 43 DEGs from this subset of six samples from our data do not paint as clear of a picture, and most of them unexpectedly adhere to the $x=y$ line in the treatment plots. (PNG $579 \mathrm{~kb}$ )

Additional file 13: Scatterplot matrix of virus-related DEGs from our dataset, showing only replicates 7,8 , and 9 .

The 43 virus-related DEGs from our dataset superimposed as magenta dots onto all genes in the form of a scatterplot matrix. Only replicates 7, 8, and 9 are shown from both treatment groups. The data has been standardized. " $\mathrm{N}$ " represents non-inoculated control samples and "V" represents virus-treated samples. We see that, compared to the scatterplot matrices from certain clusters of the Galbraith data, the 43 DEGs from this subset of six samples from our data do not paint as clear of a picture, sometimes unexpectedly deviating from the $x=y$ line in the replicate plots and sometimes unexpectedly adhering to the $x=y$ line in the treatment plots. (PNG $565 \mathrm{~kb}$ )

Additional file 14: Scatterplot matrix of virus-related DEGs from our dataset, showing only replicates 10,11 , and 12 .

The 43 virus-related DEGs from our dataset superimposed onto all genes in the form of a scatterplot matrix. Only replicates 10,11, and 12 are shown from both treatment groups. The data has been standardized. " $\mathrm{N}$ " represents non-inoculated control samples and " $V$ " represents virus-treated samples. We see that, compared to the scatterplot matrices from certain clusters of the Galbraith data, the 43 DEGs from this subset of six samples from our data do not paint as clear of a picture, and most of them unexpectedly deviate from the $x=y$ line in the virus-related replicate plots. (PNG $587 \mathrm{~kb}$ )

Additional file 15: Parallel coordinate plots of the "tolerance" candidate DEGs.

Parallel coordinate plots of the 122 DEGs after hierarchical clustering of size four between the "tolerance" candidate DEGs. Here " $\mathrm{N}$ " represents non-inoculated control group, "V" represents treatment of virus, "C" represents high quality chestnut diet, and " $\mathrm{R}$ " represents low quality rockrose diet. The vertical red line indicates the distinction between treatment groups. We see there is considerable noise in the data (non-consistent replicate values), but that the general patterns of the DEGs follow what we expect based on our "tolerance" contrast. (PNG $1741 \mathrm{~kb}$ )

Additional file 16: Parallel coordinate plots of the "resistance" candidate DEGs.

Parallel coordinate plots of the 125 DEGs after hierarchical clustering of size four between the "resistance" candidate DEGs. Here " $\mathrm{N}$ " represents non-inoculated control group, "V" represents treatment of virus, "C" represents high quality chestnut diet, and " $\mathrm{R}$ " represents low quality rockrose diet. The vertical red line indicates the distinction between treatment groups. We see there is considerable noise in the data (non-consistent replicate values), but that the general patterns of the DEGs follow what we expect based on our "resistance" contrasts. (PNG 2014 kb)

Additional file 17: Venn diagrams comparing the virus-related DEG overlaps in the Galbraith data using our pipeline and the pipeline used by Galbraith et al.

Venn diagrams comparing the virus-related DEG overlaps of the Galbraith data from the DESeq2 bioinformatics pipelines used in the Galbraith study (labeled as "G.O.") and the DESeq2 bioinformatics pipelines used in our study (labeled as "G.R"). While we were not able to fully replicate the DEG list published in the Galbraith study, our DEG list maintained significant overlaps with their DEG list. From left to right: Total virus-related DEGs (subplot A), virus-upregulated DEGs (subplot B), control-upregulated DEGs (subplot C). (PNG $164 \mathrm{~kb}$ )

Additional file 18: Venn diagrams of main effect DEG overlaps across DESeq2, edgeR, and limma

Venn diagrams comparing DEG overlaps across DESeq2, edgeR, and limma for our diet main effect (top row), our virus main effect (middle row), and the Galbraith virus main effect (bottom row). Within a given subplot, "D" represents DESeq2, "E" represents edgeR, and " $L$ " represents limma. From left to right on top row: Total diet-related DEGs (subplot A),

chestnut-upregulated DEGs (subplot B), rockrose-upregulated DEGs (subplot C). From left to right on middle row: Total virus-related DEGs (subplot D), virus-upregulated DEGs (subplot E), control-upregulated DEGs in our data (subplot F). From left to right on bottom row: Total virus-related DEGs (subplot G), virus-upregulated DEGs (subplot H), control-upregulated DEGs in the Galbraith data (subplot I) (PNG). With the exception of the limma pipeline resulting in zero DEGs in our virus main effect analysis, we found significant overlaps between DEG lists across the different pipelines (DESeq2, edgeR, and limma). In general, DESeq2 resulted in the largest number of DEGs and limma resulted in the least number of DEGs. (PNG $537 \mathrm{~kb}$ )

Additional file 19: Analysis of correlation between DEG read counts and pathogen response metrics

Distribution of R-squared values for DEG cluster read counts and pathogen response metrics. Columns left to right: SBV titers, mortality rates, and IAPV 
titers. Rows top to bottom: Tolerance candidate DEGs, resistance candidate $D E G s$, and virus-related DEGs. Each subplot includes five boxplots which represent the R-squared value distributions for four DEG clusters and all remaining non-DEGs in the data. The top number above each boxplot represents the number of genes included. The first four boxplots also include a bottom number, which represents the Kruskal-Wallis $p$-value of the comparison of the R-squared distribution of the cluster and the R-squared distribution of the non-DEG data. (PNG $323 \mathrm{~kb}$ )

Additional file 20: Tables listing DEGs for contrasts.

Table 1: IDs of 1914 DEGs in our diet main effect. Table 2: IDs of 43 DEGs in our virus main effect. Table 3: IDs of 178 DEGs in our NR versus VR contrast. Table 4: IDs of 376 DEGs in our VC versus VR contrast. Table 5: IDs of 774 DEGs in our NC versus VR contrast. Table 6: IDs of 955 DEGs in our VC versus NR contrast. Table 7: IDs of 941 DEGs in our NC versus NR contrast. Table 8: IDs of 125 resistance candidate genes. Table 9: IDs of 122 tolerance candidate genes. (XLS $1376 \mathrm{~kb}$ )

\section{Abbreviations}

DEG: Differentially expressed gene; DWV: Deformed wing virus; GO: Gene ontology; IAPV: Israeli acute paralysis virus; NC: Non-inoculation, high quality pollen (chestnut) pollen treatment; NR: Non-inoculation, low quality (rockrose) pollen treatment; PCD: Principal component analysis; REML: Restricted maximum likelihood; SBV: Sacbrood virus; VC: IAPV-inoculated, high quality pollen (chestnut) pollen treatment; VR: IAPV-inoculated, low quality (rockrose) pollen treatment

\section{Acknowledgements}

We would like to thank Giselle Narvaez for assisting with cage experiments.

\section{Funding}

This work was supported by the United States Department of Agriculture, Agriculture and Food Research Initiative (USDA-AFRI) 2011-04894, but it had no role in the design, analysis, or interpretation of the study.

\section{Availability of data and materials}

The data discussed in this publication have been deposited in NCBI's Gene Expression Omnibus [105] and are accessible through GEO Series accession number GSE121885 (https://www.ncbi.nlm.nih.gov/geo/query/acc.cgi?acc= GSE121885). Scripts to reproduce visualizations in this paper are available online (https://github.com/lindsayrutter/HoneyBeePaper). Information about bigPint visualizations, including how to intrepret them are available online (https://lindsayrutter.github.io/bigPint). Lists of BeeBase IDs for contrast DEGs from this study are found in Additional file 20.

\section{Authors' contributions}

LR performed the bioinformatic and statistical analyses and produced the figures and tables. LR, AGD, and ALT drafted the manuscript. All authors contributed to the conception of the project, the experimental design, and the interpretation of the data. AGD and JCT carried out laboratory experiments. AGD processed samples for virus and RNA-seq. DC advised on statistical analyses and visualization. All authors revised and approved the manuscript.

\section{Ethics approval and consent to participate}

All honey bees used in this work were sampled in the United States, and no ethical use approval is required for this species in this country.

\section{Consent for publication}

Not applicable.

\section{Competing interests}

The authors declare that they have no competing interests.

\section{Publisher's Note}

Springer Nature remains neutral with regard to jurisdictional claims in published maps and institutional affiliations.

\section{Author details}

${ }^{1}$ Bioinformatics and Computational Biology Program, lowa State University, Ames 50011, IA, USA. ${ }^{2}$ Department of Microbiology, Center for Scientific Research and Higher Education of Ensenada, Ensenada 22860, Baja California,
Mexico. ${ }^{3}$ Department of Entomology and Nematology, University of Florida, Gainesville 32611, FL, USA. ${ }^{4}$ Econometrics and Business Statistics, Monash University, Clayton 3800, VIC, Australia. ${ }^{5}$ Department of Entomology, lowa State University, Ames 50011, IA, USA. ${ }^{6}$ Department of Ecology, Evolution, and Organismal Biology, lowa State University, Ames 50011, IA, USA. ${ }^{7}$ Department of Entomology, University of Illinois at Urbana-Champaign, Urbana 61801, IL, USA.

Received: 23 November 2018 Accepted: 3 May 2019

Published online: 22 May 2019

\section{References}

1. van Engelsdorp D, Evans JD, Saegerman C, Mullin C, Haubruge E, Nguyen BK, Frazier M, Frazier J, Cox-Foster D, Chen Y, Underwood R, Tarpy DR, Pettis JS. Colony collapse disorder: A descriptive study. PLoS ONE. 2009;4:6481.

2. Kulhanek K, Steinhauer N, Rennich K, Caron DM, Sagili RR, Pettis JS, Ellis JD, Wilson ME, Wilkes JT, Tarpy DR, Rose R, Lee K, Rangel J, vanEngelsdorp D. A national survey of managed honey bee 2014-2015 annual colony losses in the USA. J Apic Res. 2017;56:328-40.

3. Laurent M, Hendrikx P, Ribiere-Chabert M, Chauzat M-P. A pan-European epidemiological study on honeybee colony losses 2012-2014. Epilobee. 2016;2013:44

4. Caron D, Sagili R. Honey bee colony mortality in the Pacific Northwest: Winter 2009/2010. Am Bee J. 2011;151:73-6.

5. Bond J, Plattner K, Hunt K. Fruit and Tree Nuts Outlook: Economic Insight U.S. Pollination- Services Market. USDA: Economic Research Service Situation and Outlook FTS-357SA; 2014.

6. Gallai N, Salles J-M, Settele J, Vaissière BB. Economic valuation of the vulnerability of world agriculture confronted with pollinator decline. Ecol Econ. 2009;68:810-21.

7. Klein A-M, Vaissière BE, Cane JH, Steffan-Dewenter I, Cunningham SA, Kremen C, Tscharntke T. Importance of pollinators in changing landscapes for world crops. Proc Biol Sci. 2007;274:303-13.

8. Potts SG, Biesmeijer JC, Kremen C, Neumann P, Schweiger O, Kunin WE. Global pollinator declines: trends, impacts and drivers. Trends Ecol Evol. 2010:25:345-53.

9. Spivak M, Mader E, Vaughan M, Euliss NH. The Plight of the Bees. Environ Sci Technol. 2011:45:34-8.

10. Goulson D, Nicholls E, Botías C, Rotheray EL. Bee declines driven by combined stress from parasites, pesticides, and lack of flowers. Science. 2015;347:1255957.

11. Dolezal AG, Carrillo-Tripp J, Judd TM, Miller WA, Bonning BC, Toth AL. Interacting stressors matter: diet quality and virus infection in honeybee health. Roy Soc Open Sci. 2019;6:181803.

12. Dolezal AG, Toth AL. Feedbacks between nutrition and disease in honey bee health. Curr Opin Insect Sci. 2018;26:114-9.

13. Roulston TH, Buchmann SL. A phylogenetic reconsideration of the pollen starch-pollination correlation. Evol Ecol Res. 2000;2:627-43.

14. Stanley RG, Linskens HF. Pollen: Biology, Biochemistry, Management. Philadelphia: Springer Science \& Business Media; 1974.

15. Brodschneider R, Crailsheim K. Nutrition and health in honey bees. Apidologie. 2010;41:278-94.

16. Haydak MH. Honey bee nutrition. Annu Rev Entomol. 1970;15:143-56.

17. Crailsheim $K$, Schneider LHW, Hrassnigg N, Bühlmann G, Brosch $U$, Gmeinbauer R, Schöffmann B. Pollen consumption and utilization in worker honeybees (Apis mellifera carnica): dependence on individual age and function. J Insect Physiol. 1992;38:409-19.

18. Crailsheim K. The flow of jelly within a honeybee colony. J Comp Physiol B. 1992;162:681-9.

19. Schmidt JO. Feeding preference of Apis mellifera L. (Hymenoptera: Apidae): Individual versus mixed pollen species. J Kans Entomol Soc. 1984;57:323-7.

20. Schmidt JO, Thoenes SC, Levin MD. Survival of honey bees, Apis mellifera (Hymenoptera: Apidae), fed various pollen sources. J Econ Entomol. 1987;80:176-83.

21. Alaux C, Ducloz F, Conte DCYL. Diet effects on honeybee immunocompetence. Biol Lett. 2010;6:562-5.

22. Naug D. Nutritional stress due to habitat loss may explain recent honeybee colony collapses. Biol Conserv. 2009;142:2369-72. 
23. Engelsdorp DV, Hayes JJ, Underwood RM, Pettis J. A survey of honey bee colony losses in the U.S., fall 2007 to spring 2008. PLoS ONE. 2008;3: 4071.

24. Neumann P, Carreck NL. Honey bee colony losses. J Apicult Res. 2010;49:1-6.

25. Engelsdorp DV, Meixner MD. A historical review of managed honey bee populations in Europe and the United States and the factors that may affect them. J Invertebr Pathol. 2010;103:80-95.

26. Pasquale GD, Salignon M, Conte YL, Belzunces LP, Decourtye A, Kretzschmar A, Suchail S, Brunet J-L, Alaux C. Influence of pollen nutrition on honey bee health: Do pollen quality and diversity matter? PLOS ONE. 2013;8:72016.

27. Rosenkranz P, Aumeier P, Ziegelmann B. Biology and control of Varroa destructor. Journal of invertebrate pathology. J Invertebr Pathol. 2010;103:96-119.

28. Allen MF, Ball BV, White RF, Antoniw JF. The detection of acute paralysis virus in Varroa jacobsoni by the use of a simple indirect ELISA Apicult Res. 1986;25:100-5.

29. Yue C, Genersch E. RT-PCR analysis of deformed wing virus in honeybees (Apis mellifera) and mites (Varroa destructor). J Gen Virol. 2005:86:3419-24.

30. Weinberg KP, Madel G. The influence of the mite Varroa Jacobsoni Oud on the protein concentration and the haemolymph volume of the brood of worker bees and drones of the honey bee Apis Mellifera L. Apidologie. 1985;16:421-36.

31. Ramsey SD, Ochoa R, Bauchan G, Gulbronson C, Mowery JD, Cohen A, Lim D, Joklik J, Cicero JM, Ellis JD, Hawthorne D, vanEngelsdorp D. Varroa destructor feeds primarily on honey bee fat body tissue and not hemolymph. PNAS. 2019;116:1792-801.

32. Aronstein KA, Saldivar E, Vega R, Westmiller S, Douglas AE. How Varroa Parasitism Affects the Immunological and Nutritional Status of the Honey Bee, Apis mellifera. Insects. 2012;3:601-15.

33. Shen MQ, Cui LW, Ostiguy N, Cox-Foster D. Intricate transmission routes and interactions between picorna-like viruses (Kashmir bee virus and sacbrood virus) with the honeybee host and the parasitic varroa mite. J Gen Virol. 2005:86:2281-9.

34. Yang X, Cox-Foster D. Effects of parasitization by Varroa destructor on survivorship and physiological traits of Apis mellifera in correlation with viral incidence and microbial challenge. Parasitology. 2007;134:405-12.

35. Yang XL, Cox-Foster DL. Impact of an ectoparasite on the immunity and pathology of an invertebrate: Evidence for host immunosuppression and viral amplification. P Natl Acad Sci USA. 2005;102:7470-5.

36. Emsen B, Hamiduzzaman MM, Goodwin PH, Guzman-Novoa E. Lower virus infections in Varroa destructor-infested and uninfested brood and adult honey bees (Apis mellifera) of a low mite population growth colony compared to a high mite population growth colony. PLOS ONE. 2015:10:0118885.

37. Chen YP, Siede R. Honey bee viruses. Adv Virus Res. 2007;70:33-80.

38. Bonning BC, Miller WA. Dicistroviruses. Annu Rev Entomol. 2010;55: 129-50.

39. Maori E, Paldi N, Shafir S, Kalev H, Tsur E, Glick E, Sela I. IAPV, a bee-affecting virus associated with Colony Collapse Disorder can be silenced by dsRNA ingestion. Insect Mol Biol. 2009;18:55-60.

40. Carrillo-Tripp J, Dolezal AG, Goblirsch MJ, Miller WA, Toth AL, Bonning $B C$. In vivo and in vitro infection dynamics of honey bee viruses. Sci Rep. 2016:6:22265.

41. Chen YP, Pettis JS, Corona M, Chen WP, Li CJ, Spivak M, Visscher PK, DeGrandi-Hoffman G, Boncristiani H, Zhao Y, van Engelsdorp D, Delaplane K, Solter L, Drummond F, Kramer M, Lipkin WI, Palacios G, Hamilton MC, Smith B, Huang SK, Zheng HQ, Li JL, Zhang X, Zhou XF, Wu LY, Zhou JZ, Lee M-L, Teixeira EW, Li ZG, Evans JD. Israeli acute paralysis virus: Epidemiology, pathogenesis and implications for honey bee health. PLoS Pathog. 2014;10:1004261

42. DeGrandi-Hoffman G, Chen Y. Nutrition, immunity and viral infections in honey bees. Curr Opin Insect Sci. 2015;10:170-6.

43. DeGrandi-Hoffman $G$, Chen $Y$, Huang E, Huang MH. The effect of diet on protein concentration, hypopharyngeal gland development and virus load in worker honey bees (Apis mellifera L.) J Insect Physiol. 2010;56:1184-91.

44. Le Conte Y, BRUNET J-L, McDonnell C, Dussaubat C, Alaux C. Interactions between risk factors in honey bees. In: Sammataro D,
Yoder JA, editors. Honey bee colony health. Challenges and sustainable solutions. Boca Raton: CRC Press; 2012. p. 215-22.

45. Annoscia D, Zanni V, Galbraith D, Quirici A, Grozinger C, Bortolomeazzi R, Nazzi F. Elucidating the mechanisms underlying the beneficial health effects of dietary pollen on honey bees (Apis mellifera) infested by Varroa mite ectoparasites. Sci Rep. 2017;7:6258.

46. Nazzi F, Pennacchio F. Honey bee antiviral immune barriers as affected by multiple stress factors: A novel paradigm to interpret colony health decline and collapse. Viruses. 2018;10:159.

47. Medzhitov R, Schneider DS, Soares MP. Disease tolerance as a defense strategy. Science. 2012;335:936-41.

48. Alaux C, Dantec C, Parrinello H, Conte YL. Nutrigenomics in honey bees: digital gene expression analysis of pollen's nutritive effects on healthy and varroa-parasitized bees. BMC Genomics. 2011;12:496.

49. Galbraith DA, Yang X, Niño EL, Yi S, Grozinger C. Parallel epigenomic and transcriptomic responses to viral infection in honey bees (Apis mellifera). PLoS Pathog. 2015;11:1004713.

50. Brutscher LM, Daughenbaugh KF, Flenniken ML. Virus and dsRNA-triggered transcriptional responses reveal key components of honey bee antiviral defense. Sci Rep. 2017;7:6448.

51. Chen YP, Pettis JS, Corona M, Chen WP, Li CJ, Spivak M, Visscher PK DeGrandi-Hoffman G, Boncristiani H, Zhao Y, vanEngelsdorp D, Delaplane K, Solter L, Drummond F, Kramer M, Lipkin WI, Palacios G, Hamilton MC, Smith B, Huang SK, Zheng HQ, Li JL, Zhang X, Zhou AF, Wu LY, Zhou JZ, Lee M-L, Teixeira EW, Li ZG, Evans JD. Israeli Acute Paralysis Virus: Epidemiology, pathogenesis and implications for honey bee health. PLoS Pathog. 2014;10:1004261.

52. Ryabov EV, Fannon JM, Moore JD, Wood GR, Evans DJ. The Iflaviruses Sacbrood virus and Deformed wing virus evoke different transcriptional responses in the honeybee which may facilitate their horizontal or vertical transmission. PeerJ. 2016;4:1591.

53. Vincent D, Poeschl Y, Gogol-Döring A, Alaux C, Annoscia D, Aurori C, Barribeau SM, et al. Unity in defence: honeybee workers exhibit conserved molecular responses to diverse pathogens. BMC Genomics. 2017; 18:207.

54. Hemphill W, Rivera O, Talbert M. RNA-Sequencing of Drosophila melanogaster head tissue on high-sugar and high-fat diets. G3: Genes, Genomes, Genetics. 2018;8:279-90.

55. Nazario-Yepiz NO, Loustalot-Laclette MR, Carpinteyro-Ponce J, Abreu-Goodger C, Markow TA. Transcriptional responses of ecologically diverse Drosophila species to larval diets differing in relative sugar and protein ratios. PLoS ONE. 2017;12:0183007.

56. Mirth CK, Tang HY, Makohon-Moore SC, Salhadar S, Gokhale RH, Warner RD, Koyama T, Riddiford LM, Shingleton AW. Juvenile hormone regulates body size and perturbs insulin signaling in Drosophila. Proc Natl Acad Sci. 2014;25:201313058.

57. O'Neal ST, Swale DR, Anderson TD. ATP-sensitive inwardly rectifying potassium channel regulation of viral infections in honey bees. Sci Rep. 2017;7:8668

58. O'Neal ST, Brewster CC, Bloomquist JR, Anderson TD. Amitraz and its metabolite modulate honey bee cardiac function and tolerance to viral infection. J Invertebr Pathol. 2017;149:119-26.

59. Grozinger CM, Flenniken ML. Bee viruses: Ecology, pathogenicity, and impacts. Ann Rev Entomol. 2019;64:205-26.

60. Xu J, Cherry S. Viruses and antiviral immunity in Drosophila. Dev Comp Immunol. 2014:42:67-84.

61. Swevers L, Liu J, Smagghe G. Defense Mechanisms against Viral Infection in Drosophila: RNAi and Non-RNAi. Viruses. 2018;10:230.

62. McMenamin AJ, Daughenbaugh KF, Parekh F, Pizzorno MC, Flenniken ML. Honey Bee and Bumble Bee Antiviral Defense. Viruses. 2018;10:395.

63. Page RE, Laidlaw HH. Full sisters and supersisters: A terminological paradigm. Anim Behav. 1988;36:944-5.

64. Sherman PW, Seeley TD, Reeve HK. Parasites, pathogens, and polyandry in social Hymenoptera. Am Nat. 1988;131:602-10.

65. Crozier RH, Page RE. On being the right size: Male contributions and multiple mating in social Hymenoptera. Behav Ecol Sociobiol. 1985;18: 105-15

66. Mattila HR, Seeley TD. Genetic diversity in honey bee colonies enhances productivity and fitness. Science. 2007;317:362-4

67. Tarpy DR. Genetic diversity within honeybee colonies prevents severe infections and promotes colony growth. Proc R Soc Lond B. 2003;270: 99-103. 
68. Brodschneider R, Arnold G, Hrassnigg N, Crailsheim K. Does patriline composition change over a honey bee queen's lifetime?,. Insects. 2012;3:857-69.

69. Hansen KD, Brenner SE, Dudoit S. Biases in Illumina transcriptome sequencing caused by random hexamer priming. Nucleic Acids Res. 2010;38:131.

70. Oshlack A, Robinson MD, Young MD. From RNA-seq reads to differential expression results. Genome Biol. 2010;11:220.

71. Mclntyre LM, Lopiano KK, Morse AM, Amin V, Oberg AL, Young LJ, Nuzhdin SV. RNAseq: Technical variability and sampling. BMC Genomics. 2011;12:293.

72. Rutter L, Cook D. bigPint: Big multivariate data plotted interactively. 2018. https://github.com/lindsayrutter/bigPint. Accessed 22 Nov 2018.

73. Thissen D, Steinberg L, Kuang D. Quick and easy implementation of the Benjamini-Hochberg procedure for controlling the false positive rate in multiple comparisons. J Educ Behav Stat. 2002;27:77-83.

74. Traynor KS, Conte YL, Page RE. Age matters: pheromone profiles of larvae differentially influence foraging behaviour in the honeybee, Apis mellifera. Anim Behav. 2015;99:1-8.

75. Consortium H. B. G. S. Finding the missing honey bee genes: lessons learned from a genome upgrade. BMC Genomics. 2014;15:86.

76. Elsik CG, Tayal A, Diesh CM, Unni DR, Emery ML, Nguyen HN, Hagen DE. Hymenoptera Genome Database: integrating genome annotations in HymenopteraMine. Nucleic Acids Res. 2016;4:793-800.

77. Wu TD, Reeder J, Lawrence M, Becker G, Brauer MJ. GMAP and GSNAP for genomic sequence alignment: Enhancements to speed, accuracy, and functionality. Methods Mol Biol. 2016;1418:283-334.

78. Love MI, Huber W, Anders S. Moderated estimation of fold change and dispersion for RNA-seq data with DESeq2. Genome Biol. 2014;15:550

79. Robinson MD, McCarthy DJ, Smyth GK. edger: a bioconductor package for differential expression analysis of digital gene expression data. Bioinformatics. 2010;26:139-40.

80. Ritchie ME, Phipson B, Wu D, Hu Y, Law CW, Shi W, Smyth GK. limma powers differential expression analyses for rna-sequencing and microarray studies. Nucleic Acids Res. 2015;43(7):47.

81. R Development Core Team. R: A Language and Environment for Statistical Computing. Vienna: R Foundation for Statistical Computing; 2008. http://www.R-project.org. R Foundation for Statistical Computing. ISBN 3-900051-07-0.

82. Benjamini $Y$, Hochberg Y. Controlling the false discovery rate: A practical and powerful approach to multiple testing. J Roy Stat Soc Ser B (Methodol). 1995:57:289-300

83. Larsson J. eulerr: Area-Proportional Euler and Venn Diagrams with Ellipses. 2018. https://cran.r-project.org/package=eulerr. Accessed 1 Nov 2017. R package version 4.0.0.

84. IT Jolliffe. Principal Component Analysis. Berlin, Heidelberg: Springer; 2002.

85. Inselberg A. The plane with parallel coordinates. Vis Comput. 1985;1: 69-91.

86. WS Cleveland. Visualizing Data. Summit: Hobart Press; 1993.

87. Cook D, Hofmann H, Lee E, Yang H, Nikolau B, Wurtele E. Exploring gene expression data, using plots. J Data Sci. 2007;5:151-82.

88. Chandrasekhar T, Thangavel K, Elayaraja E. Effective Clustering Algorithms for Gene Expression Data. Int J Comput Appl. 2011;32:4

89. de Souto D, de Araujo M, Costa I, Soares R, Ludermir T, Schliep A Comparative Study on Normalization Procedures for Cluster Analysis of Gene Expression Datasets. Int Jt Conf Neural Netw. 2008;2792-8.

90. Huang DW, Sherman BT, Lempicki R. Systematic and integrative analysis of large gene lists using DAVID bioinformatics resources. Nat Protoc. 2009;4:44-57.

91. Huang DW, Sherman BT, Lempicki RA. Bioinformatics enrichment tools: paths toward the comprehensive functional analysis of large gene lists. Nucleic Acids Res. 2009;37:1-13.

92. Supek F, Bošnjak M, Škunca N, Šmuc T. REVIGO summarizes and visualizes long lists of Gene Ontology terms. PLoS ONE. 2011;6:21800

93. Merkling SH, Overheul GJ, van Mierlo JT, Arends D, Gilissen C, van Rij RP. The heat shock response restricts virus infection in Drosophila. Sci Rep. 2015;5:12758.

94. Dostert C, Jouanguy E, Irving P, Troxler L, Galiana D, Hetru C, Hoffmann JA, Imler J-L. The JAK-STAT signaling pathway is required but not sufficient for the antiviral response of Drosophila. Nat Immunol. 2005;6:946-53.
95. Flenniken ML, Andino R. Non-specific dsRNA-mediated antiviral response in the honey bee. PLOS ONE. 2013;8:77263.

96. Carval D, Ferriere R. A unified model for the coevolution of resistance, tolerance, and virulence. Evolution. 2010;64:2988-3009.

97. Miller CVL, Cotter SC. Resistance and tolerance: The role of nutrients on pathogen dynamics and infection outcomes in an insect host. J Anim Ecol. 2017;87:500-10.

98. Moret Y. Trans-generational immune priming: Specific enhancement of the antimicrobial immune response in the mealworm beetle, Tenebrio molitor. Proc Roy Soc B: Biol Sci. 2006;273:1399-405.

99. Mauricio R, Rausher MD, Burdick DS. Variation in the defense strategies of plants: are resistance and tolerance mutually exclusive?,. Ecology. 1997;78:1301-10.

100. Fornoni J, Nunez-Farfan J, Valverde PL, Rausher MD. Evolution of mixed plant defense allocation against natural enemies. Evolution. 2004;58: 1685-95.

101. Restif O, Koella JC. Shared control of epidemiological traits in a coevolutionary model of host-parasite interactions. Am Nat. 2003;161: 827-36.

102. Chambers MC, Schneider DS. Balancing resistance and infection tolerance through metabolic means. PNAS. 2012;109:13886-7.

103. Xu J, Grant G, Sabin LR, Gordesky-Gold B, Yasunaga A, Tudor M, Cherry S. Transcriptional pausing controls a rapid antiviral innate immune response in Drosophila. Cell Host Microbe. 2012;12:531-43.

104. Johnson BR, Atallah J, Plachetzki DC. The importance of tissue specificity for RNA-seq: highlighting the errors of composite structure extractions. BMC Genomics. 2013;14:586

105. Edgar R, Domrachev M, Lash AE. Gene Expression Omnibus: NCBI gene expression and hybridization array data repository. Nucleic Acids Res. 2002;30:207-10.

106. Schlicker A, Domingues FS, Rahnenfuhrer J, Lengauer T. A new measure for functional similarity of gene products based on Gene Ontology. BMC Bioinformatics. 2006;7:302.
Ready to submit your research? Choose BMC and benefit from:

- fast, convenient online submission

- thorough peer review by experienced researchers in your field

- rapid publication on acceptance

- support for research data, including large and complex data types

- gold Open Access which fosters wider collaboration and increased citations

- maximum visibility for your research: over 100M website views per year

At $\mathrm{BMC}$, research is always in progress.

Learn more biomedcentral.com/submission 